\title{
GENERALIZED MINIMUM DISTANCE FUNCTIONS
}

\author{
MANUEL GONZÁLEZ-SARABIA, JOSÉ MARTÍNEZ-BERNAL, RAFAEL H. VILLARREAL, \\ AND CARLOS E. VIVARES
}

\begin{abstract}
Using commutative algebra methods we study the generalized minimum distance function (gmd function) and the corresponding generalized footprint function of a graded ideal in a polynomial ring over a field. The number of solutions that a system of homogeneous polynomials has in any given finite set of projective points is expressed as the degree of a graded ideal. If $\mathbb{X}$ is a set of projective points over a finite field and $I$ is its vanishing ideal, we show that the gmd function and the Vasconcelos function of $I$ are equal to the $r$-th generalized Hamming weight of the corresponding Reed-Muller-type code $C_{\mathbb{X}}(d)$ of degree $d$. We show that the generalized footprint function of $I$ is a lower bound for the $r$-th generalized Hamming weight of $C_{\mathbb{X}}(d)$. Then we present some applications to projective nested cartesian codes. To give applications of our lower bound to algebraic coding theory, we show an interesting integer inequality. Then we show an explicit formula and a combinatorial formula for the second generalized Hamming weight of an affine cartesian code.
\end{abstract}

\section{INTRODUCTION}

Let $S=K\left[t_{1}, \ldots, t_{s}\right]=\oplus_{d=0}^{\infty} S_{d}$ be a polynomial ring over a field $K$ with the standard grading and let $I \neq(0)$ be a graded ideal of $S$. In this work we extend the scope of [25] by considering generalized footprint and minimum distance functions. Given $d, r \in \mathbb{N}_{+}$, let $\mathcal{F}_{d, r}$ be the set:

$$
\mathcal{F}_{d, r}:=\left\{\left\{f_{1}, \ldots, f_{r}\right\} \subset S_{d} \mid \bar{f}_{1}, \ldots, \bar{f}_{r} \text { are linearly independent over } K,\left(I:\left(f_{1}, \ldots, f_{r}\right)\right) \neq I\right\},
$$

where $\bar{f}=f+I$ is the class of $f$ modulo $I$, and $\left(I:\left(f_{1}, \ldots, f_{r}\right)\right)=\left\{g \in S \mid g f_{i} \in I, \forall i\right\}$ is referred to as an ideal quotient or colon ideal.

We denote the degree of $S / I$ by $\operatorname{deg}(S / I)$. The function $\delta_{I}: \mathbb{N}_{+} \times \mathbb{N}_{+} \rightarrow \mathbb{Z}$ given by

$$
\delta_{I}(d, r):= \begin{cases}\operatorname{deg}(S / I)-\max \left\{\operatorname{deg}(S /(I, F)) \mid F \in \mathcal{F}_{d, r}\right\} & \text { if } \mathcal{F}_{d, r} \neq \emptyset \\ \operatorname{deg}(S / I) & \text { if } \mathcal{F}_{d, r}=\emptyset\end{cases}
$$

is called the generalized minimum distance function of $I$, or simply the gmd function of $I$. If $r=1$ one obtains the minimum distance function of $I$ [25]. To compute $\delta_{I}(d, r)$ is a difficult problem. One of our aims is to introduce lower bounds for $\delta_{I}(d, r)$ which are easier to compute.

Fix a monomial order $\prec$ on $S$. Let in $\prec(I)$ be the initial ideal of $I$ and let $\Delta_{\prec}(I)$ be the footprint of $S / I$ consisting of all the standard monomials of $S / I$ with respect to $\prec$. The footprint of $S / I$ is also called the Gröbner éscalier of $I$. Given integers $d, r \geq 1$, let $\mathcal{M}_{\prec, d, r}$ be the set of all subsets $M$ of $\Delta_{\prec}(I)_{d}:=\Delta_{\prec}(I) \cap S_{d}$ with $r$ distinct elements such that $\left(\operatorname{in}_{\prec}(I):(M)\right) \neq \operatorname{in}_{\prec}(I)$. The generalized footprint function of $I$, denoted $\mathrm{fp}_{I}$, is the function $\mathrm{fp}_{I}: \mathbb{N}_{+} \times \mathbb{N}_{+} \rightarrow \mathbb{Z}$ given by

$$
\operatorname{fp}_{I}(d, r):= \begin{cases}\operatorname{deg}(S / I)-\max \left\{\operatorname{deg}\left(S /\left(\operatorname{in}_{\prec}(I), M\right)\right) \mid M \in \mathcal{M}_{\prec, d, r}\right\} & \text { if } \mathcal{M}_{\prec, d, r} \neq \emptyset \\ \operatorname{deg}(S / I) & \text { if } \mathcal{M}_{\prec, d, r}=\emptyset .\end{cases}
$$

2010 Mathematics Subject Classification. Primary 13P25; Secondary 14G50, 94B27, 11 T71.

Key words and phrases. Reed-Muller-type codes, minimum distance, vanishing ideal, degree, Hilbert function.

The first author was supported by COFAA-IPN and SNI, Mexico. The second and third author were supported by SNI, Mexico. The fourth author was supported by a scholarship from CONACyT, Mexico. 
If $r=1$ one obtains the footprint function of $I$ that was studied in 28 from a theoretical point of view (see [25, 26] for some applications). The footprint of vanishing ideals of finite sets of affine points was used in the works of Geil [9] and Carvalho [3] to study affine Reed-Mullertype codes. Long before these two papers appeared the footprint was used by Geil in connection with all kinds of codes (including one-point algebraic geometric codes); see [10, 11, 12] and the references therein.

The definition of $\delta_{I}(d, r)$ was motivated by the notion of generalized Hamming weight of a linear code [19, 21, 37]. For convenience we recall this notion. Let $K=\mathbb{F}_{q}$ be a finite field and let $C$ be a $[m, k]$ linear code of length $m$ and dimension $k$, that is, $C$ is a linear subspace of $K^{m}$ with $k=\operatorname{dim}_{K}(C)$. Let $1 \leq r \leq k$ be an integer. Given a subcode $D$ of $C$ (that is, $D$ is a linear subspace of $C)$, the support $\chi(D)$ of $D$ is the set of non-zero positions of $D$, that is,

$$
\chi(D):=\left\{i \mid \exists\left(a_{1}, \ldots, a_{m}\right) \in D, a_{i} \neq 0\right\} .
$$

The $r$-th generalized Hamming weight of $C$, denoted $\delta_{r}(C)$, is the size of the smallest support of an $r$-dimensional subcode. Generalized Hamming weights have received a lot of attention; see [3, 6, 9, 15, 18, 20, 30, 32, 35, 37, 38, 39] and the references therein. The study of these weights is related to trellis coding, $t$-resilient functions, and was motivated by some applications from cryptography [37].

The minimum distance of projective Reed-Muller-type codes has been studied using Gröbner bases and commutative algebra techniques; see [3, 4, 9, 13, 25, 31] and the references therein. In this work we extend these techniques to study the $r$-th generalized Hamming weight of projective Reed-Muller-type codes. These linear codes are constructed as follows.

Let $K=\mathbb{F}_{q}$ be a finite field with $q$ elements, let $\mathbb{P}^{s-1}$ be a projective space over $K$, and let $\mathbb{X}$ be a subset of $\mathbb{P}^{s-1}$. The vanishing ideal of $\mathbb{X}$, denoted $I(\mathbb{X})$, is the ideal of $S$ generated by the homogeneous polynomials that vanish at all points of $\mathbb{X}$. The Hilbert function of $S / I(\mathbb{X})$ is denoted by $H_{\mathbb{X}}(d)$. We can write $\mathbb{X}=\left\{\left[P_{1}\right], \ldots,\left[P_{m}\right]\right\} \subset \mathbb{P}^{s-1}$ with $m=|\mathbb{X}|$. Here we assume that the first non-zero entry of each $\left[P_{i}\right]$ is 1 . In the special case that $\mathbb{X}$ has the form $[X \times\{1\}]$ for some $X \subset \mathbb{F}_{q}^{s-1}$, we assume that the $s$-th entry of each $\left[P_{i}\right]$ is 1 .

Fix a degree $d \geq 1$. There is a $K$-linear map given by

$$
\mathrm{ev}_{d}: S_{d} \rightarrow K^{m}, \quad f \mapsto\left(f\left(P_{1}\right), \ldots, f\left(P_{m}\right)\right) .
$$

The image of $S_{d}$ under $\mathrm{ev}_{d}$, denoted by $C_{\mathbb{X}}(d)$, is called a projective Reed-Muller-type code of degree $d$ on $\mathbb{X}[7,16]$. The points in $\mathbb{X}$ are often called evaluation points in the algebraic coding context. The parameters of the linear code $C_{\mathbb{X}}(d)$ are:

(a) length: $|\mathbb{X}|$,

(b) dimension: $\operatorname{dim}_{K} C_{\mathbb{X}}(d)$,

(c) $r$-th generalized Hamming weight: $\delta_{\mathbb{X}}(d, r):=\delta_{r}\left(C_{\mathbb{X}}(d)\right)$.

The contents of this paper are as follows. In Section 2 we present some of the results and terminology that will be needed throughout the paper. If $F$ is a finite set of homogeneous polynomials of $S \backslash\{0\}$ and $V_{\mathbb{X}}(F)$ is the set of zeros or projective variety of $F$ in $\mathbb{X}$, over a finite field, we show a degree formula for counting the number of points in $\mathbb{X}$ that are not in $V_{\mathbb{X}}(F)$ :

$$
\left|\mathbb{X} \backslash V_{\mathbb{X}}(F)\right|=\left\{\begin{array}{cc}
\operatorname{deg}(S /(I(\mathbb{X}):(F))) & \text { if }(I(\mathbb{X}):(F)) \neq I(\mathbb{X}), \\
\operatorname{deg}(S / I(\mathbb{X})) & \text { if }(I(\mathbb{X}):(F))=I(\mathbb{X}),
\end{array}\right.
$$

and a degree formula for counting the zeros of $F$ in $\mathbb{X}$ (Lemmas 3.2 and 3.4). These degree formulas turn out to be useful in order to prove one of our main results (Theorem 4.9). 
If $\mathbb{X}$ is a finite set of projective points over a finite field and $I(\mathbb{X})$ is its vanishing ideal, we show that $\delta_{I(\mathbb{X})}(d, r)$ is the $r$-th generalized Hamming weight $\delta_{\mathbb{X}}(d, r)$ of the corresponding Reed-Muller-type code $C_{\mathbb{X}}(d)$ (Theorem 4.5). We introduce the Vasconcelos function $\vartheta_{I}(d, r)$ of a graded ideal $I$ (Definition 4.4) and show that $\vartheta_{I(\mathbb{X})}(d, r)$ is also equal to $\delta_{\mathbb{X}}(d, r)$ (Theorem 4.5). These two abstract algebraic formulations of $\delta_{\mathbb{X}}(d, r)$ gives us a new tool to study generalized Hamming weights. One of our main results shows that $\operatorname{fp}_{I(\mathbb{X})}(d, r)$ is a lower bound for $\delta_{\mathbb{X}}(d, r)$ (Theorem 4.9). The footprint matrix $\left(\mathrm{fp}_{I(\mathbb{X})}(d, r)\right)$ and the weight matrix $\left(\delta_{\mathbb{X}}(d, r)\right)$ of $I(\mathbb{X})$ are the matrices of size $\operatorname{reg}(S / I(\mathbb{X})) \times \operatorname{deg}(S / I(\mathbb{X}))$ whose $(d, r)$-entries are $f_{I(\mathbb{X})}(d, r)$ and $\delta_{\mathbb{X}}(d, r)$, respectively (see Remark 4.6). In certain cases the $r$-th columns of these two matrices are equal (Example 6.3. Theorem 9.5). The entries of each row of the weight matrix $\left(\delta_{\mathbb{X}}(d, r)\right.$ ) form an increasing sequence until they stabilize [37. For parameterized codes the entries of each column of the weight matrix $\left(\delta_{\mathbb{X}}(d, r)\right)$ form a decreasing sequence until they stabilize [14, Theorem 12].

In Section [5] we introduce projective nested cartesian codes [4], a type of evaluation codes that generalize the classical projective Reed-Muller codes [22, 27, 33. It is an interesting open problem to find an explicit formula for the minimum distance of a projective nested cartesian code. Using footprints and the integer inequality of Lemma 5.3 we show a uniform upper bound for the number of zeros in a projective nested cartesian set $\mathcal{X}$ for a family of homogeneous polynomials of fixed degree $d$, where $d$ is close to the regularity of the ideal $I(\mathcal{X})$ (Theorem 5.5). For projective spaces, this upper bound agrees with the classical upper bound of Sørensen and Serre [33, p. 1569].

As an application of our methods using generalized minimum distance functions we were able to find a simple counterexample to a conjecture of Carvalho, Lopez-Neumann and López [4], 25. Conjecture 6.2] (Example 6.1). In Sections 6 and 7 we show some examples and implementations in Macaulay2 [17] that illustrate how some of our results can be used in practice. A finite set of generators for the vanishing ideal of a projective space, over a finite field, was found by Mercier and Rolland [27, Corollaire 2.1]. More generally, for the vanishing ideal of a projective nested cartesian set a finite set of generators was determined in [4, Lemma 2.4]. These results are especially useful for computational purposes (Examples 6.1 and 6.4).

To show some other applications to algebraic coding theory we prove the following interesting and non-trivial inequality.

Theorem 8.5 Let $d \geq 1$ and $1 \leq e_{1} \leq \cdots \leq e_{m}$ be integers. Suppose $1 \leq a_{i} \leq e_{i}$ and $1 \leq b_{i} \leq e_{i}$, for $i=1, \ldots, m$, are integers such that $d=\sum_{i} a_{i}=\sum_{i} b_{i}$ and $a \neq b$. Then

$$
\pi(a, b) \geq\left(\sum_{i=1}^{m} a_{i}-\sum_{i=k+1}^{m} e_{i}-(k-2)\right) e_{k+1} \cdots e_{m}-e_{k+2} \cdots e_{m}
$$

for $k=1, \ldots, m-1$, where $\pi(a, b)=\prod_{i=1}^{m} a_{i}+\prod_{i=1}^{m} b_{i}-\prod_{i=1}^{m} \min \left(a_{i}, b_{i}\right)$.

We give two more applications. The first is the following explicit formula for the second generalized Hamming weight of an affine cartesian code.

Theorem 9.3 Let $A_{i}, i=1, \ldots, s-1$, be subsets of $\mathbb{F}_{q}$ and let $\mathbb{X} \subset \mathbb{P}^{s-1}$ be the projective set $\mathbb{X}=\left[A_{1} \times \cdots \times A_{s-1} \times\{1\}\right]$. If $d_{i}=\left|A_{i}\right|$ for $i=1, \ldots, s-1$ and $2 \leq d_{1} \leq \cdots \leq d_{s-1}$, then

$$
\delta_{\mathbb{X}}(d, 2)=\left\{\begin{array}{cl}
\left(d_{k+1}-\ell+1\right) d_{k+2} \cdots d_{s-1}-d_{k+3} \cdots d_{s-1} & \text { if } k<s-3, \\
\left(d_{k+1}-\ell+1\right) d_{k+2} \cdots d_{s-1}-1 & \text { if } k=s-3, \\
d_{s-1}-\ell+1 & \text { if } k=s-2,
\end{array}\right.
$$

where $0 \leq k \leq s-2$ and $\ell$ are integers, $d=\sum_{i=1}^{k}\left(d_{i}-1\right)+\ell$, and $1 \leq \ell \leq d_{k+1}-1$. 
Using this result one recover the case when $\mathbb{X}$ is a projective torus in $\mathbb{P}^{s-1}$ [14, Theorem 18] (Corollary 9.4). The second applications of this paper gives a combinatorial formula for the second generalized Hamming weight of an affine cartesian code, which is quite different from the corresponding formula of [1, Theorem 5.4], and show that in this case the second generalized Hamming weight is equal to the second generalized footprint.

Theorem 9.5 Let $\mathcal{P}_{d}$ be the set of all pairs $(a, b), a, b$ in $\mathbb{N}^{s}, a=\left(a_{i}\right), b=\left(b_{i}\right)$, such that $a \neq b$, $d=\sum_{i} a_{i}=\sum_{i} b_{i}, 1 \leq a_{i}, b_{i} \leq d_{i}-1$ for $i=1, \ldots, n, n:=s-1, a_{i} \neq 0$ and $b_{j} \neq 0$ for some $1 \leq i, j \leq n$. If $\mathbb{X}=\left[A_{1} \times \cdots \times A_{n} \times\{1\}\right]$, with $A_{i} \subset \mathbb{F}_{q}, d_{i}=\left|A_{i}\right|$, and $2 \leq d_{1} \leq \cdots \leq d_{n}$, then

$$
\mathrm{fp}_{I(\mathbb{X})}(d, 2)=\delta_{\mathbb{X}}(d, 2)=\min \left\{P(a, b) \mid(a, b) \in \mathcal{P}_{d}\right\} \quad \text { for } d \leq \sum_{i=1}^{n}\left(d_{i}-1\right),
$$

where $P(a, b)=\prod_{i=1}^{n}\left(d_{i}-a_{i}\right)+\prod_{i=1}^{n}\left(d_{i}-b_{i}\right)-\prod_{i=1}^{n} \min \left\{d_{i}-a_{i}, d_{i}-b_{i}\right\}$.

In case the set of evaluation points $\mathbb{X}$ lie on an affine algebraic variety over a finite field $\mathbb{F}_{q}$, the work done by Heijnen and Pellikaan [18, though formulated in a different language, relates footprints and generalized Hamming weights and introduce methods to study affine cartesian codes (cf. [18, Section 7]). These methods were used in [1] to determine the generalized Hamming weights of these codes.

There is a nice combinatorial formula to compute the generalized Hamming weights of $q$-ary Reed-Muller codes [18, Theorem 3.14], and there is an easy to evaluate formula for the second generalized Hamming weight of a projective torus [14, Theorem 18]. There is also a recent expression for the $r$-th generalized Hamming weight of an affine cartesian code [1, Theorem 5.4], which depends on the $r$-th monomial in ascending lexicographic order of a certain family of monomials. It is an interesting problem to find alternative, easy to evaluate formulas for the $r$-th generalized Hamming weight of an affine cartesian code.

For all unexplained terminology and additional information we refer to [2, 5, 8] (for the theory of Gröbner bases, commutative algebra, and Hilbert functions), and [24, 35] (for the theory of error-correcting codes and linear codes).

\section{Preliminaries}

In this section we present some of the results that will be needed throughout the paper and introduce some more notation. All results of this section are well-known. To avoid repetitions, we continue to employ the notations and definitions used in Section 1.

Generalized Hamming weights. Let $K=\mathbb{F}_{q}$ be a finite field and let $C$ be a $[m, k]$ linear code of length $m$ and dimension $k$.

The $r$-th generalized Hamming weight of $C$, denoted $\delta_{r}(C)$, is the size of the smallest support of an $r$-dimensional subcode, that is,

$$
\delta_{r}(C):=\min \left\{|\chi(D)|: D \text { is a linear subcode of } C \text { with } \operatorname{dim}_{K}(D)=r\right\} .
$$

The weight hierarchy of $C$ is the sequence $\left(\delta_{1}(C), \ldots, \delta_{k}(C)\right)$. The integer $\delta_{1}(C)$ is called the minimum distance of $C$ and is denoted by $\delta(C)$. According to [37, Theorem 1, Corollary 1] the weight hierarchy is an increasing sequence

$$
1 \leq \delta_{1}(C)<\cdots<\delta_{k}(C) \leq m,
$$

and $\delta_{r}(C) \leq m-k+r$ for $r=1, \ldots, k$. For $r=1$ this is the Singleton bound for the minimum distance. Notice that $\delta_{r}(C) \geq r$.

Recall that the support $\chi(\beta)$ of a vector $\beta \in K^{m}$ is $\chi(K \beta)$, that is, $\chi(\beta)$ is the set of non-zero entries of $\beta$. 
Lemma 2.1. Let $D$ be a subcode of $C$ of dimension $r \geq 1$. If $\beta_{1}, \ldots, \beta_{r}$ is a $K$-basis for $D$ with $\beta_{i}=\left(\beta_{i, 1}, \ldots, \beta_{i, m}\right)$ for $i=1, \ldots, r$, then $\chi(D)=\cup_{i=1}^{r} \chi\left(\beta_{i}\right)$ and the number of elements of $\chi(D)$ is the number of non-zero columns of the matrix:

$$
\left[\begin{array}{ccccc}
\beta_{1,1} & \cdots & \beta_{1, i} & \cdots & \beta_{1, m} \\
\beta_{2,1} & \cdots & \beta_{2, i} & \cdots & \beta_{2, m} \\
\vdots & \cdots & \vdots & \cdots & \vdots \\
\beta_{r, 1} & \cdots & \beta_{r, i} & \cdots & \beta_{r, m}
\end{array}\right] .
$$

Commutative algebra. Let $I \neq(0)$ be a graded ideal of $S$ of Krull dimension $k$. The Hilbert function of $S / I$ is:

$$
H_{I}(d):=\operatorname{dim}_{K}\left(S_{d} / I_{d}\right), \quad d=0,1,2, \ldots,
$$

where $I_{d}=I \cap S_{d}$. By a theorem of Hilbert [34, p. 58], there is a unique polynomial $h_{I}(x) \in \mathbb{Q}[x]$ of degree $k-1$ such that $H_{I}(d)=h_{I}(d)$ for $d \gg 0$. The degree of the zero polynomial is -1 .

The degree or multiplicity of $S / I$ is the positive integer

$$
\operatorname{deg}(S / I):= \begin{cases}(k-1) ! \lim _{d \rightarrow \infty} H_{I}(d) / d^{k-1} & \text { if } k \geq 1, \\ \operatorname{dim}_{K}(S / I) & \text { if } k=0 .\end{cases}
$$

We will use the following multi-index notation: for $a=\left(a_{1}, \ldots, a_{s}\right) \in \mathbb{N}^{s}$, set $t^{a}:=t_{1}^{a_{1}} \cdots t_{s}^{a_{s}}$. The multiplicative group of the field $K$ is denoted by $K^{*}$. As usual ht $(I)$ will denote the height of the ideal $I$. By the dimension of $I$ (resp. $S / I$ ) we mean the Krull dimension of $S / I$. The Krull dimension of $S / I$ is denoted by $\operatorname{dim}(S / I)$.

One of the most useful and well-known facts about the degree is its additivity:

Proposition 2.2. (Additivity of the degree [29, Proposition 2.5]) If $I$ is an ideal of $S$ and $I=\mathfrak{q}_{1} \cap \cdots \cap \mathfrak{q}_{m}$ is an irredundant primary decomposition, then

$$
\operatorname{deg}(S / I)=\sum_{\operatorname{ht}\left(\mathfrak{q}_{i}\right)=\operatorname{ht}(I)} \operatorname{deg}\left(S / \mathfrak{q}_{i}\right)
$$

If $F \subset S$, the ideal quotient of $I$ with respect to $(F)$ is given by $(I:(F))=\{h \in S \mid h F \subset I\}$. An element $f$ is called a zero-divisor of $S / I$ if there is $\overline{0} \neq \bar{a} \in S / I$ such that $f \bar{a}=\overline{0}$, and $f$ is called regular on $S / I$ if $f$ is not a zero-divisor. Thus $f$ is a zero-divisor if and only if $(I: f) \neq I$. An associated prime of $I$ is a prime ideal $\mathfrak{p}$ of $S$ of the form $\mathfrak{p}=(I: f)$ for some $f$ in $S$.

Theorem 2.3. [36, Lemma 2.1.19, Corollary 2.1.30] If $I$ is an ideal of $S$ and $I=\mathfrak{q}_{1} \cap \cdots \cap \mathfrak{q}_{m}$ is an irredundant primary decomposition with $\operatorname{rad}\left(\mathfrak{q}_{i}\right)=\mathfrak{p}_{i}$, then the set of zero-divisors $\mathcal{Z}(S / I)$ of $S / I$ is equal to $\bigcup_{i=1}^{m} \mathfrak{p}_{i}$, and $\mathfrak{p}_{1}, \ldots, \mathfrak{p}_{m}$ are the associated primes of $I$.

Definition 2.4. The regularity of $S / I$, denoted $\operatorname{reg}(S / I)$, is the least integer $r_{0} \geq 0$ such that $H_{I}(d)$ is equal to $h_{I}(d)$ for $d \geq r_{0}$.

The footprint of an ideal. Let $\prec$ be a monomial order on $S$ and let $(0) \neq I \subset S$ be an ideal. If $f$ is a non-zero polynomial in $S$, the leading monomial of $f$ is denoted by $\operatorname{in}_{\prec}(f)$. The initial ideal of $I$, denoted by $\operatorname{in}_{\prec}(I)$, is the monomial ideal given by

$$
\operatorname{in}_{\prec}(I)=\left(\left\{\operatorname{in}_{\prec}(f) \mid f \in I\right\}\right) .
$$

A monomial $t^{a}$ is called a standard monomial of $S / I$, with respect to $\prec$, if $t^{a}$ is not in the ideal in $\prec(I)$. A polynomial $f$ is called standard if $f \neq 0$ and $f$ is a $K$-linear combination of standard monomials. The set of standard monomials, denoted $\Delta_{\prec}(I)$, is called the footprint of $S / I$. The image of the standard polynomials of degree $d$, under the canonical map $S \mapsto S / I$, 
$x \mapsto \bar{x}$, is equal to $S_{d} / I_{d}$, and the image of $\Delta_{\prec}(I)$ is a basis of $S / I$ as a $K$-vector space. This is a classical result of Macaulay (for a modern approach see [5, Chapter 5]). In particular, if $I$ is graded, then $H_{I}(d)$ is the number of standard monomials of degree $d$.

Lemma 2.5. [3, p. 2] Let $I \subset S$ be an ideal generated by $\mathcal{G}=\left\{g_{1}, \ldots, g_{r}\right\}$, then

$$
\Delta_{\prec}(I) \subset \Delta_{\prec}\left(\operatorname{in}_{\prec}\left(g_{1}\right), \ldots, \operatorname{in}_{\prec}\left(g_{r}\right)\right) .
$$

Vanishing ideal of a finite set. The projective space of dimension $s-1$ over the field $K$ is denoted $\mathbb{P}^{s-1}$. It is usual to denote the equivalence class of $\alpha$ by $[\alpha]$.

For a given a subset $\mathbb{X} \subset \mathbb{P}^{s-1}$ define $I(\mathbb{X})$, the vanishing ideal of $\mathbb{X}$, as the ideal generated by the homogeneous polynomials in $S$ that vanish at all points of $\mathbb{X}$, and given a graded ideal $I \subset S$ define its zero set relative to $\mathbb{X}$ as

$$
V_{\mathbb{X}}(I)=\{[\alpha] \in \mathbb{X} \mid f(\alpha)=0 \forall f \in I \text { homogeneous }\} .
$$

In particular, if $f \in S$ is homogeneous, the zero set $V_{\mathbb{X}}(f)$ of $f$ is the set of all $[\alpha] \in \mathbb{X}$ such that $f(\alpha)=0$, that is $V_{\mathbb{X}}(f)$ is the set of zeros of $f$ in $\mathbb{X}$.

Lemma 2.6. Let $\mathbb{X}$ be a finite subset of $\mathbb{P}^{s-1}$, let $[\alpha]$ be a point in $\mathbb{X}$ with $\alpha=\left(\alpha_{1}, \ldots, \alpha_{s}\right)$ and $\alpha_{k} \neq 0$ for some $k$, and let $I_{[\alpha]}$ be the vanishing ideal of $[\alpha]$. Then $I_{[\alpha]}$ is a prime ideal,

$$
I_{[\alpha]}=\left(\left\{\alpha_{k} t_{i}-\alpha_{i} t_{k} \mid k \neq i \in\{1, \ldots, s\}\right), \operatorname{deg}\left(S / I_{[\alpha]}\right)=1,\right.
$$

$\operatorname{ht}\left(I_{[\alpha]}\right)=s-1$, and $I(\mathbb{X})=\bigcap_{[\beta] \in \mathbb{X}} I_{[\beta]}$ is the primary decomposition of $I(\mathbb{X})$.

Definition 2.7. The set $\mathbb{T}=\left\{\left[\left(x_{1}, \ldots, x_{s}\right)\right] \in \mathbb{P}^{s-1} \mid x_{i} \in K^{*}, \forall i\right\}$ is called a projective torus.

\section{Computing the number of POINTS OF A VARIETy}

In this section we give a degree formula to compute the number of solutions of a system of homogeneous polynomials over any given finite set of points in a projective space over a field.

Lemma 3.1. Let $\mathbb{X}$ be a finite subset of $\mathbb{P}^{s-1}$ over a field $K$. If $F=\left\{f_{1}, \ldots, f_{r}\right\}$ is a set of homogeneous polynomials of $S \backslash\{0\}$, then $V_{\mathbb{X}}(F)=\emptyset$ if and only if $(I(\mathbb{X}):(F))=I(\mathbb{X})$.

Proof. $\Rightarrow$ ) We proceed by contradiction assuming that $I(\mathbb{X}) \subsetneq(I(\mathbb{X}):(F))$. Pick a homogeneous polynomial $g$ such that $g f_{i} \in I(\mathbb{X})$ for all $i$ and $g \notin I(\mathbb{X})$. Then there is $[\alpha]$ in $\mathbb{X}$ such that $g(\alpha) \neq 0$. Thus $f_{i}(\alpha)=0$ for all $i$, that is, $[\alpha] \in V_{\mathbb{X}}(F)$, a contradiction.

$\Leftarrow)$ We can write $\mathbb{X}=\left\{\left[P_{1}\right], \ldots,\left[P_{m}\right]\right\}$ and $I(\mathbb{X})=\cap_{i=1}^{m} \mathfrak{p}_{i}$, where $\mathfrak{p}_{i}$ is equal to $I_{\left[P_{i}\right]}$, the vanishing ideal of $\left[P_{i}\right]$. We proceed by contradiction assuming that $V_{\mathbb{X}}(F) \neq \emptyset$. Pick $\left[P_{i}\right]$ in $V_{\mathbb{X}}(F)$. For simplicity of notation assume that $i=1$. Notice that $\left(\mathfrak{p}_{1}:(F)\right)=(1)$. Therefore

$$
\bigcap_{i=1}^{m} \mathfrak{p}_{i}=I(\mathbb{X})=(I(\mathbb{X}):(F))=\bigcap_{i=1}^{m}\left(\mathfrak{p}_{i}:(F)\right)=\bigcap_{i=2}^{m}\left(\mathfrak{p}_{i}:(F)\right) \subset \mathfrak{p}_{1} .
$$

Hence $\mathfrak{p}_{i} \subset\left(\mathfrak{p}_{i}:(F)\right) \subset \mathfrak{p}_{1}$ for some $i \geq 2$, see [36, p. 74]. Thus $\mathfrak{p}_{i}=\mathfrak{p}_{1}$, a contradiction.

An ideal $I \subset S$ is called unmixed if all its associated primes have the same height, and $I$ is called radical if $I$ is equal to its radical. The radical of $I$ is denoted by $\operatorname{rad}(I)$.

Lemma 3.2. Let $\mathbb{X}$ be a finite subset of $\mathbb{P}^{s-1}$ over a field $K$ and let $I(\mathbb{X}) \subset S$ be its vanishing ideal. If $F=\left\{f_{1}, \ldots, f_{r}\right\}$ is a set of homogeneous polynomials of $S \backslash\{0\}$, then

$$
\left|\mathbb{X} \backslash V_{\mathbb{X}}(F)\right|=\left\{\begin{array}{cl}
\operatorname{deg}(S /(I(\mathbb{X}):(F))) & \text { if }(I(\mathbb{X}):(F)) \neq I(\mathbb{X}), \\
\operatorname{deg}(S / I(\mathbb{X})) & \text { if }(I(\mathbb{X}):(F))=I(\mathbb{X})
\end{array}\right.
$$


Proof. Let $\left[P_{1}\right], \ldots,\left[P_{m}\right]$ be the points of $\mathbb{X}$ with $m=|\mathbb{X}|$, and let $[P]$ be a point in $\mathbb{X}$ with $P=\left(\alpha_{1}, \ldots, \alpha_{s}\right)$ and $\alpha_{k} \neq 0$ for some $k$. Then the vanishing ideal $I_{[P]}$ of $[P]$ is a prime ideal of height $s-1$,

$$
I_{[P]}=\left(\left\{\alpha_{k} t_{i}-\alpha_{i} t_{k} \mid k \neq i \in\{1, \ldots, s\}\right), \operatorname{deg}\left(S / I_{[P]}\right)=1,\right.
$$

and $I(\mathbb{X})=\bigcap_{i=1}^{m} I_{\left[P_{i}\right]}$ is a primary decomposition (see Lemma 2.6).

Assume that $(I(\mathbb{X}):(F)) \neq I(\mathbb{X})$. We set $I=I(\mathbb{X})$ and $\mathfrak{p}_{i}=I_{\left[P_{i}\right]}$ for $i=1, \ldots, m$. Notice that $\left(\mathfrak{p}_{j}: f_{i}\right)=(1)$ if and only if $f_{i} \in \mathfrak{p}_{j}$ if and only if $f_{i}\left(P_{j}\right)=0$. Then

$$
(I:(F))=\bigcap_{i=1}^{r}\left(I: f_{i}\right)=\left(\bigcap_{f_{1}\left(P_{j}\right) \neq 0} \mathfrak{p}_{j}\right) \bigcap \cdots \bigcap\left(\bigcap_{f_{r}\left(P_{j}\right) \neq 0} \mathfrak{p}_{j}\right)=\bigcap_{\left[P_{j}\right] \notin V_{\mathbb{X}}(F)} \mathfrak{p}_{j} .
$$

Therefore, by the additivity of the degree of Proposition 2.2 , we get that $\operatorname{deg}(S /(I:(F)))$ is equal to $\left|\mathbb{X} \backslash V_{\mathbb{X}}(F)\right|$. If $(I(\mathbb{X}):(F))=I(\mathbb{X})$, then $V_{\mathbb{X}}(F)=\emptyset$ (see Lemma 3.1) ). Thus $\left|V_{\mathbb{X}}(F)\right|=0$ and the required formula follows because $|\mathbb{X}|=\operatorname{deg}(S / I(\mathbb{X}))$.

Lemma 3.3. Let $I \subset S$ be a radical unmixed graded ideal. If $F=\left\{f_{1}, \ldots, f_{r}\right\}$ is a set of homogeneous polynomials of $S \backslash\{0\},(I:(F)) \neq I$, and $\mathcal{A}$ is the set of all associated primes of $S / I$ that contain $F$, then $\operatorname{ht}(I)=\operatorname{ht}(I, F), \mathcal{A} \neq \emptyset$ and

$$
\operatorname{deg}(S /(I, F))=\sum_{\mathfrak{p} \in \mathcal{A}} \operatorname{deg}(S / \mathfrak{p}) .
$$

Proof. As $I \subsetneq(I:(F))$, there is $g \in S \backslash I$ such that $g(F) \subset I$. Hence the ideal $(F)$ is contained in the set of zero-divisors of $S / I$. Thus, by Theorem 2.3 and since $I$ is unmixed, $(F)$ is contained in an associated prime ideal $\mathfrak{p}$ of $S / I$ of height $h t(I)$. Thus $I \subset(I, F) \subset \mathfrak{p}$, and consequently $\mathrm{ht}(I)=\mathrm{ht}(I, F)$. Therefore the set of associated primes of $(I, F)$ of height equal to ht $(I)$ is not empty and is equal to $\mathcal{A}$. There is an irredundant primary decomposition

$$
(I, F)=\mathfrak{q}_{1} \cap \cdots \cap \mathfrak{q}_{n} \cap \mathfrak{q}_{n+1}^{\prime} \cap \cdots \cap \mathfrak{q}_{t}^{\prime},
$$

where $\operatorname{rad}\left(\mathfrak{q}_{i}\right)=\mathfrak{p}_{\mathfrak{i}}, \mathcal{A}=\left\{\mathfrak{p}_{1}, \ldots, \mathfrak{p}_{n}\right\}$, and $\mathrm{ht}\left(\mathfrak{q}_{i}^{\prime}\right)>\operatorname{ht}(I)$ for $i>n$. We may assume that the associated primes of $S / I$ are $\mathfrak{p}_{1}, \ldots, \mathfrak{p}_{m}$ with $n \leq m$. Since $I$ is a radical ideal, we get that $I=\cap_{i=1}^{m} \mathfrak{p}_{i}$. Next we show the following equality:

$$
\mathfrak{p}_{1} \cap \cdots \cap \mathfrak{p}_{m}=\mathfrak{q}_{1} \cap \cdots \cap \mathfrak{q}_{n} \cap \mathfrak{q}_{n+1}^{\prime} \cap \cdots \cap \mathfrak{q}_{t}^{\prime} \cap \mathfrak{p}_{n+1} \cap \cdots \cap \mathfrak{p}_{m} .
$$

The inclusion " $\supset$ " is clear because $\mathfrak{q}_{i} \subset \mathfrak{p}_{i}$ for $i=1, \ldots, n$. The inclusion " $\subset$ " follows by noticing that the right hand side of Eq. (3.2) is equal to $(I, f) \cap \mathfrak{p}_{n+1} \cap \cdots \cap \mathfrak{p}_{m}$, and consequently it contains $I=\cap_{i=1}^{m} \mathfrak{p}_{i}$. Notice that $\operatorname{rad}\left(\mathfrak{q}_{j}^{\prime}\right)=\mathfrak{p}_{j}^{\prime} \not \subset \mathfrak{p}_{i}$ for all $i, j$ and $\mathfrak{p}_{j} \not \subset \mathfrak{p}_{i}$ for $i \neq j$. Hence localizing Eq. (3.2) at the prime ideal $\mathfrak{p}_{i}$ for $i=1, \ldots, n$, we get that $\mathfrak{p}_{i}=I_{\mathfrak{p}_{i}} \cap S=\left(\mathfrak{q}_{i}\right)_{\mathfrak{p}_{i}} \cap S=\mathfrak{q}_{i}$ for $i=1, \ldots, n$. Using Eq. (3.1), together with the additivity of the degree of Proposition 2.2, the required equality follows.

Lemma 3.4. Let $\mathbb{X}$ be a finite subset of $\mathbb{P}^{s-1}$ over a field $K$ and let $I(\mathbb{X}) \subset S$ be its vanishing ideal. If $F=\left\{f_{1}, \ldots, f_{r}\right\}$ is a set of homogeneous polynomials of $S \backslash\{0\}$, then the number of points of $V_{\mathbb{X}}(F)$ is given by

$$
\left|V_{\mathbb{X}}(F)\right|=\left\{\begin{array}{cl}
\operatorname{deg}(S /(I(\mathbb{X}), F)) & \text { if }(I(\mathbb{X}):(F)) \neq I(\mathbb{X}) \\
0 & \text { if }(I(\mathbb{X}):(F))=I(\mathbb{X})
\end{array}\right.
$$

Proof. Let $\left[P_{1}\right], \ldots,\left[P_{m}\right]$ be the points of $\mathbb{X}$ with $m=|\mathbb{X}|$. The vanishing ideal $I_{\left[P_{i}\right]}$ of $\left[P_{i}\right]$ is a prime ideal of height $s-1, \operatorname{deg}\left(S / I_{\left[P_{i}\right]}\right)=1$, and $I(\mathbb{X})=\bigcap_{i=1}^{m} I_{\left[P_{i}\right]}$ (see Lemma [2.6). 
Assume that $(I(\mathbb{X}):(F)) \neq I(\mathbb{X})$. Let $\mathcal{A}$ be the set of all $I_{\left[P_{i}\right]}$ that contain the set $F$. Notice that $f_{j} \in I_{\left[P_{i}\right]}$ if and only if $f_{j}\left(P_{i}\right)=0$. Then $\left[P_{i}\right]$ is in $V_{\mathbb{X}}(F)$ if and only if $F \subset I_{\left[P_{i}\right]}$. Thus $\left[P_{i}\right]$ is in $V_{\mathbb{X}}(F)$ if and only if $I_{\left[P_{i}\right]}$ is in $\mathcal{A}$. Hence, by Lemma 3.3, we get

$$
\left|V_{\mathbb{X}}(F)\right|=\sum_{\left[P_{i}\right] \in V_{\mathbb{X}}(F)} \operatorname{deg}\left(S / I_{\left[P_{i}\right]}\right)=\sum_{F \subset I_{\left[P_{i}\right]}} \operatorname{deg}\left(S / I_{\left[P_{i}\right]}\right)=\operatorname{deg}(S /(I(\mathbb{X}), F)) .
$$

Assume that $(I(\mathbb{X}): F)=I(\mathbb{X})$. Then, by Lemma 3.1, $V_{\mathbb{X}}(f)=\emptyset$ and $\left|V_{\mathbb{X}}(f)\right|=0$.

Proposition 3.5. If $\mathbb{X}$ is a finite subset of $\mathbb{P}^{s-1}$, then

$$
\operatorname{deg}(S / I(\mathbb{X}))=\operatorname{deg}(S /(I(\mathbb{X}), F))+\operatorname{deg}(S /(I(\mathbb{X}):(F)))
$$

Proof. It follows from Lemmas 3.2 and 3.4 .

\section{Generalized minimum distance function of A GRAded ideal}

In this part we study the generalized minimum distance function of a graded ideal. To avoid repetitions, we continue to employ the notations and definitions used in Sections 1 and 2 .

Lemma 4.1. Let $I \subset S$ be an unmixed graded ideal and let $\prec$ be a monomial order. If $F$ is a finite set of homogeneous polynomials of $S$ and $(I:(F)) \neq I$, then

$$
\operatorname{deg}(S /(I, F)) \leq \operatorname{deg}\left(S /\left(\operatorname{in}_{\prec}(I), \operatorname{in}_{\prec}(F)\right)\right) \leq \operatorname{deg}(S / I),
$$

and $\operatorname{deg}(S /(I, F))<\operatorname{deg}(S / I)$ if $I$ is an unmixed radical ideal and $(F) \not \subset I$.

Proof. To simplify notation we set $J=(I, F), L=\left(\operatorname{in}_{\prec}(I), \operatorname{in}_{\prec}(F)\right)$, and $F=\left\{f_{1}, \ldots, f_{r}\right\}$. We denote the Krull dimension of $S / I$ by $\operatorname{dim}(S / I)$. Recall that $\operatorname{dim}(S / I)=\operatorname{dim}(S)-\operatorname{ht}(I)$. First we show that $S / J$ and $S / L$ have Krull dimension equal to $\operatorname{dim}(S / I)$. As $I \subsetneq(I: F)$, all elements of $F$ are zero divisors of $S / I$. Hence, as $I$ is unmixed, there is an associated prime ideal $\mathfrak{p}$ of $S / I$ such that $(F) \subset \mathfrak{p}$ and $\operatorname{dim}(S / I)=\operatorname{dim}(S / \mathfrak{p})$. Since $I \subset J \subset \mathfrak{p}$, we get that $\operatorname{dim}(S / J)$ is $\operatorname{dim}(S / I)$. Since $S / I$ and $S / \operatorname{in}_{\prec}(I)$ have the same Hilbert function, and so does $S / \mathfrak{p}$ and $S / \operatorname{in}_{\prec}(\mathfrak{p})$, we obtain

$$
\operatorname{dim}\left(S / \operatorname{in}_{\prec}(I)\right)=\operatorname{dim}(S / I)=\operatorname{dim}(S / \mathfrak{p})=\operatorname{dim}\left(S / \operatorname{in}_{\prec}(\mathfrak{p})\right) .
$$

Hence, taking heights in the inclusions $\operatorname{in}_{\prec}(I) \subset L \subset \operatorname{in}_{\prec}(\mathfrak{p})$, we obtain $\operatorname{ht}(I)=\operatorname{ht}(L)$.

Pick a Gröbner basis $\mathcal{G}=\left\{g_{1}, \ldots, g_{r}\right\}$ of $I$. Then $J$ is generated by $\mathcal{G} \cup F$ and by Lemma 2.5 one has the inclusions

$$
\begin{aligned}
& \Delta_{\prec}(J)=\Delta_{\prec}(I, F) \subset \Delta_{\prec}\left(\operatorname{in}_{\prec}\left(g_{1}\right), \ldots, \operatorname{in}_{\prec}\left(g_{r}\right), \operatorname{in}_{\prec}(F)\right)= \\
& \quad \Delta_{\prec}\left(\operatorname{in}_{\prec}(I), \operatorname{in}_{\prec}(F)\right)=\Delta_{\prec}(L) \subset \Delta_{\prec}\left(\operatorname{in}_{\prec}\left(g_{1}\right), \ldots, \operatorname{in}_{\prec}\left(g_{r}\right)\right)=\Delta_{\prec}(I) .
\end{aligned}
$$

Thus $\Delta_{\prec}(J) \subset \Delta_{\prec}(L) \subset \Delta_{\prec}(I)$. Recall that $H_{I}(d)$, the Hilbert function of $I$ at $d$, is the number of standard monomials of degree $d$. Hence $H_{J}(d) \leq H_{L}(d) \leq H_{I}(d)$ for $d \geq 0$. If $\operatorname{dim}(S / I)$ is equal to 0 , then

$$
\operatorname{deg}(S / J)=\sum_{d \geq 0} H_{J}(d) \leq \operatorname{deg}(S / L)=\sum_{d \geq 0} H_{L}(d) \leq \operatorname{deg}(S / I)=\sum_{d \geq 0} H_{I}(d) .
$$

Assume now that $\operatorname{dim}(S / I) \geq 1$. By a theorem of Hilbert [34, p. 58], $H_{J}, H_{L}, H_{I}$ are polynomial functions of degree equal to $k=\operatorname{dim}(S / I)-1$ (see [2, Theorem 4.1.3]). Thus

$$
k ! \lim _{d \rightarrow \infty} H_{J}(d) / d^{k} \leq k ! \lim _{d \rightarrow \infty} H_{L}(d) / d^{k} \leq k ! \lim _{d \rightarrow \infty} H_{I}(d) / d^{k},
$$

that is, $\operatorname{deg}(S / J) \leq \operatorname{deg}(S / L) \leq \operatorname{deg}(S / I)$. 
If $I$ is an unmixed radical ideal and $(F) \not \subset I$, then there is at least one minimal prime that does not contains $(F)$. Hence, by Lemma 3.3, it follows that $\operatorname{deg}(S /(I, F))<\operatorname{deg}(S / I)$.

Corollary 4.2. Let $\mathbb{X}$ be a finite subset of $\mathbb{P}^{s-1}$, let $I(\mathbb{X}) \subset S$ be its vanishing ideal, and let $\prec$ be a monomial order. If $F$ is a finite set of homogeneous polynomials of $S$ and $(I(\mathbb{X}):(F)) \neq I(\mathbb{X})$, then

$$
\left|V_{\mathbb{X}}(F)\right|=\operatorname{deg}(S /(I(\mathbb{X}), F)) \leq \operatorname{deg}\left(S /\left(\operatorname{in}_{\prec}(I(\mathbb{X})), \operatorname{in}_{\prec}(F)\right)\right) \leq \operatorname{deg}(S / I(\mathbb{X})),
$$

and $\operatorname{deg}(S /(I(\mathbb{X}), F))<\operatorname{deg}(S / I(\mathbb{X}))$ if $(F) \not \subset I(\mathbb{X})$.

Proof. It follows from Lemmas 3.4 and 4.1 .

Lemma 4.3. Let $\mathbb{X}=\left\{\left[P_{1}\right], \ldots,\left[P_{m}\right]\right\}$ be a finite subset of $\mathbb{P}^{s-1}$ and let $D$ be a linear subspace of $C_{\mathbb{X}}(d)$ of dimension $r \geq 1$. The following hold.

(i) There are $\bar{f}_{1}, \ldots, \bar{f}_{r}$ linearly independent elements of $S_{d} / I_{d}$ such that $D=\oplus_{i=1}^{r} K \beta_{i}$, where $\beta_{i}$ is $\left(f_{i}\left(P_{1}\right), \ldots, f_{i}\left(P_{m}\right)\right)$, and the support $\chi(D)$ of $D$ is equal to $\cup_{i=1}^{r} \chi\left(\beta_{i}\right)$.

(ii) $|\chi(D)|=\left|\mathbb{X} \backslash V_{\mathbb{X}}\left(f_{1}, \ldots, f_{r}\right)\right|$.

(iii) $\delta_{r}\left(C_{\mathbb{X}}(d)\right)=\min \left\{\left|\mathbb{X} \backslash V_{\mathbb{X}}(F)\right|: F=\left\{f_{i}\right\}_{i=1}^{r} \subset S_{d},\left\{\bar{f}_{i}\right\}_{i=1}^{r}\right.$ linearly independent over $\left.K\right\}$.

Proof. (i): This part follows from Lemma 2.1 and using that the evaluation map ev ${ }_{d}$ induces an isomorphism between $S_{d} / I_{d}$ and $C_{\mathbb{X}}(d)$.

(ii): Consider the matrix $A$ with rows $\beta_{1}, \ldots, \beta_{r}$. Notice that the $i$-th column of $A$ is not zero if and only if $\left[P_{i}\right]$ is in $\mathbb{X} \backslash V_{\mathbb{X}}\left(f_{1}, \ldots, f_{r}\right)$. It suffices to observe that the number of non-zero columns of $A$ is $|\chi(D)|$ (see Lemma 2.1).

(iii): This follows from part (ii) and using the definition of the $r$-th generalized Hamming weight of $C_{\mathbb{X}}(d)$ (see Section 2).

Definition 4.4. If $I \subset S$ is a graded ideal, the Vasconcelos function of $I$ is the function $\vartheta_{I}: \mathbb{N}_{+} \times \mathbb{N}_{+} \rightarrow \mathbb{N}$ given by

$$
\vartheta_{I}(d, r):= \begin{cases}\min \left\{\operatorname{deg}(S /(I:(F))) \mid F \in \mathcal{F}_{d, r}\right\} & \text { if } \mathcal{F}_{d, r} \neq \emptyset \\ \operatorname{deg}(S / I) & \text { if } \mathcal{F}_{d, r}=\emptyset\end{cases}
$$

Theorem 4.5. Let $K$ be a field and let $\mathbb{X}$ be a finite subset of $\mathbb{P}^{s-1}$. If $|\mathbb{X}| \geq 2$ and $\delta_{\mathbb{X}}(d, r)$ is the $r$-th generalized Hamming weight of $C_{\mathbb{X}}(d)$, then

$$
\delta_{\mathbb{X}}(d, r)=\delta_{I(\mathbb{X})}(d, r)=\vartheta_{I(\mathbb{X})}(d, r) \text { for } d \geq 1 \text { and } 1 \leq r \leq H_{I(\mathbb{X})}(d),
$$

and $\delta_{\mathbb{X}}(d, r)=r$ for $d \geq \operatorname{reg}(S / I(\mathbb{X}))$.

Proof. If $\mathcal{F}_{d, r}=\emptyset$, then using Lemmas 3.2, 3.4, and 4.3 we get that $\delta_{\mathbb{X}}(d, r), \delta_{I(\mathbb{X})}(d, r)$, and $\vartheta_{I(\mathbb{X})}(d, r)$ are equal to $\operatorname{deg}(S / I(\mathbb{X}))=|\mathbb{X}|$. Assume that $\mathcal{F}_{d, r} \neq \emptyset$ and set $I=I(\mathbb{X})$. Using Lemma 4.3 and the formula for $V_{\mathbb{X}}(F)$ of Lemma 3.4, we obtain

$$
\begin{aligned}
& \delta_{\mathbb{X}}(d, r) \stackrel{[4.3}{=} \min \left\{\left|\mathbb{X} \backslash V_{\mathbb{X}}(F)\right|: F \in \mathcal{F}_{d, r}\right\} \stackrel{\sqrt{3.4}}{=}|\mathbb{X}|-\max \left\{\operatorname{deg}(S /(I, F)) \mid F \in \mathcal{F}_{d, r}\right\} \\
&= \operatorname{deg}(S / I)-\max \left\{\operatorname{deg}(S /(I, F)) \mid F \in \mathcal{F}_{d, r}\right\}=\delta_{I}(d, r), \text { and } \\
& \delta_{\mathbb{X}}(d, r) \stackrel{\sqrt[4.3]{=}}{\stackrel{4}{=}} \min \left\{\left|\mathbb{X} \backslash V_{\mathbb{X}}(F)\right|: F \in \mathcal{F}_{d, r}\right\} \stackrel{\sqrt[3.2]{=}}{=} \min \left\{\operatorname{deg}(S /(I:(F))) \mid F \in \mathcal{F}_{d, r}\right\}=\vartheta_{I}(d, r) .
\end{aligned}
$$

In these equalities we used the fact that $\operatorname{deg}(S / I(\mathbb{X}))=|\mathbb{X}|$. As $H_{I}(d)=|\mathbb{X}|$ for $d \geq \operatorname{reg}(S / I)$, using the generalized Singleton bound for the generalized Hamming weight and the fact that the weight hierarchy is an increasing sequence we obtain that $\delta_{\mathbb{X}}(d, r)=r$ for $d \geq \operatorname{reg}(S / I(\mathbb{X}))$ (see [37, Theorem 1, Corollary 1]). 
Remark 4.6. Let $\mathbb{X}$ be a finite set of projective points over a field $K$. The following hold.

(a) $r \leq \delta_{\mathbb{X}}(d, r) \leq|\mathbb{X}|$ for $d \geq 1$ and $1 \leq r \leq H_{I(\mathbb{X})}(d)$. This follows from the fact that the weight hierarchy is an increasing sequence (see [37, Theorem 1]).

(b) If $d \geq \operatorname{reg}(S / I(\mathbb{X}))$, then $C_{\mathbb{X}}(d)=K^{|\mathbb{X}|}$ and $\delta_{\mathbb{X}}(d, r)=r$ for $1 \leq r \leq|\mathbb{X}|$.

(c) If $C_{\mathbb{X}}(d)$ is non-degenerate, i.e., for each $1 \leq i \leq|\mathbb{X}|$ there is $\alpha \in C_{\mathbb{X}}(d)$ whose $i$-th entry is non-zero, then $\delta_{\mathbb{X}}\left(d, H_{\mathbb{X}}(d)\right)=|\mathbb{X}|$.

(d) If $r>H_{I(\mathbb{X})}(d)$, then $\mathcal{F}_{d, r}=\emptyset$ and $\delta_{I(\mathbb{X})}(d, r)=|\mathbb{X}|$.

Lemma 4.7. Let $\prec$ be a monomial order, let $I \subset S$ be an ideal, let $F=\left\{f_{1}, \ldots, f_{r}\right\}$ be a set of polynomial of $S$ of positive degree, and let $\operatorname{in}_{\prec}(F)=\left\{\operatorname{in}_{\prec}\left(f_{1}\right), \ldots, \operatorname{in}_{\prec}\left(f_{r}\right)\right\}$ be the set of initial terms of $F$. If $\left(\operatorname{in}_{\prec}(I):\left(\operatorname{in}_{\prec}(F)\right)\right)=\operatorname{in}_{\prec}(I)$, then $(I:(F))=I$.

Proof. Let $g$ be a polynomial of $(I:(F))$, that is, $g f_{i} \in I$ for $i=1, \ldots, r$. It suffices to show that $g \in I$. Pick a Gröbner basis $g_{1}, \ldots, g_{n}$ of $I$. Then, by the division algorithm [5, Theorem 3 , p. 63], we can write $g=\sum_{i=1}^{n} h_{i} g_{i}+h$, where $h=0$ or $h$ is a finite sum of monomials not in $\operatorname{in}_{\prec}(I)=\left(\operatorname{in}_{\prec}\left(g_{1}\right), \ldots, \operatorname{in}_{\prec}\left(g_{n}\right)\right)$. We need only show that $h=0$. If $h \neq 0$, then $h f_{i}$ is in $I$ and $\operatorname{in}_{\prec}(h) \operatorname{in}_{\prec}\left(f_{i}\right)$ is in the ideal $\operatorname{in}_{\prec}(I)$ for $i=1, \ldots, r$. Hence $\operatorname{in}_{\prec}(h)$ is in $\left(\operatorname{in}_{\prec}(I):\left(\operatorname{in}_{\prec}(F)\right)\right)$. Therefore, by hypothesis, in $\prec(h)$ is in the ideal $\operatorname{in}_{\prec}(I)$, a contradiction.

Let $\prec$ be a monomial order and let $\mathcal{F}_{\prec, d, r}$ be the set of all subsets $F=\left\{f_{1}, \ldots, f_{r}\right\}$ of $S_{d}$ such that $(I:(F)) \neq I, f_{i}$ is a standard polynomial for all $i, \bar{f}_{1}, \ldots, \bar{f}_{r}$ are linearly independent over the field $K$, and $\operatorname{in}_{\prec}\left(f_{1}\right), \ldots$, in $_{\prec}\left(f_{r}\right)$ are distinct monomials. Let $F=\left\{f_{1}, \ldots, f_{r}\right\}$ be a set of standard polynomials. It is not hard to see that $\bar{f}_{1}, \ldots, \bar{f}_{r}$ are linearly independent over the field $K$ if in $\prec\left(f_{1}\right), \ldots$, in $_{\prec}\left(f_{r}\right)$ are distinct monomials.

The next result is useful for computations with Macaulay2 [17] (see Procedure 7.1).

Proposition 4.8. The generalized minimum distance function of I is given by

$$
\delta_{I}(d, r)= \begin{cases}\operatorname{deg}(S / I)-\max \left\{\operatorname{deg}(S /(I, F)) \mid F \in \mathcal{F}_{\prec, d, r}\right\} & \text { if } \mathcal{F}_{\prec, d, r} \neq \emptyset \\ \operatorname{deg}(S / I) & \text { if } \mathcal{F}_{\prec, d, r}=\emptyset .\end{cases}
$$

Proof. Take $F=\left\{f_{1}, \ldots, f_{r}\right\}$ in $\mathcal{F}_{d, r}$. By the division algorithm any $f_{i}$ can be written as $f_{i}=p_{i}+h_{i}$, where $p_{i}$ is in $I_{d}$ and $h_{i}$ is a $K$-linear combination of standard monomials of degree $d$. Setting $H=\left\{h_{1}, \ldots, h_{r}\right\}$, notice that $(I:(F))=(I:(H)),(I, F)=(I, H), \bar{f}_{i}=\bar{h}_{i}$ for $i=1, \ldots, r$. Thus $H \in \mathcal{F}_{d, r}$, that is, we may assume that $f_{1}, \ldots, f_{r}$ are standard polynomials. Setting $K F=K f_{1}+\cdots+K f_{r}$, we claim that there is a set $G=\left\{g_{1}, \ldots, g_{r}\right\}$ consisting of homogeneous standard polynomials of $S / I$ of degree $d$ such that $K F=K G$, in $\prec\left(g_{1}\right), \ldots$, in $_{\prec}\left(g_{r}\right)$ distinct monomials, and $\operatorname{in}_{\prec}\left(f_{i}\right) \succeq \operatorname{in}_{\prec}\left(g_{i}\right)$ for all $i$. We proceed by induction on $r$. The case $r=1$ is clear. Assume that $r>1$. Permuting the $f_{i}$ 's if necessary we may assume that $\operatorname{in}_{\prec}\left(f_{1}\right) \succeq \cdots \succeq \operatorname{in}_{\prec}\left(f_{r}\right)$. If $\operatorname{in}_{\prec}\left(f_{1}\right) \succ$ in $_{\prec}\left(f_{2}\right)$, the claim follows applying the induction hypothesis to $f_{2}, \ldots, f_{r}$. If $\operatorname{in}_{\prec}\left(f_{1}\right)=\operatorname{in}_{\prec}\left(f_{2}\right)$, there is $k \geq 2$ such that $\operatorname{in}_{\prec}\left(f_{1}\right)=\operatorname{in}_{\prec}\left(f_{i}\right)$ for $i \leq k$ and $\operatorname{in}_{\prec}\left(f_{1}\right) \succ \operatorname{in}_{\prec}\left(f_{i}\right)$ for $i>k$. We set $h_{i}=f_{1}-f_{i}$ for $i=2, \ldots, k$ and $h_{i}=f_{i}$ for $i=k+1, \ldots, r$. Notice that $\operatorname{in}_{\prec}\left(f_{1}\right) \succ h_{i}$ for $i \geq 2$ and that $h_{2}, \ldots, h_{r}$ are standard monomials of degree $d$ which are linearly independent over $K$. Hence the claim follows applying the induction hypothesis to $H=\left\{h_{2}, \ldots, h_{r}\right\}$. The required expression for $\delta_{I}(d, r)$ follows readily using Theorem 4.5 . 
Let $I$ be a graded ideal, let $\Delta_{\prec}^{p}(I)_{d}$ be the set of standard polynomials of $S / I$ of degree $d$, and let $\mathcal{F}_{\prec, d, r}^{p}$ be the set of all subsets of $\Delta_{\prec}^{p}(I)_{d}$ with $r$ elements. If $K=\mathbb{F}_{q}$ is a finite field, then

$$
\left|\Delta_{\prec}^{p}(I)_{d}\right|=q^{H_{I}(d)}-1 \text { and }\left|\mathcal{F}_{\prec, d, r}^{p}\right|=\left(\begin{array}{c}
q^{H_{I}(d)}-1 \\
r
\end{array}\right) .
$$

Thus computing $\delta_{I}(d, r)$ is very hard because one has to determine which of the polynomials in $\mathcal{F}_{\prec, d, r}^{p}$ are in $\mathcal{F}_{\prec, d, r}$, and then compute the corresponding degrees. To compute $\operatorname{fp}_{I}(d, r)$ is much simpler because we only need to determine the set $\Delta_{\prec}(I)_{d, r}$ of all subsets of $\Delta_{\prec}(I)_{d}$ with $r$ elements, and one has

$$
\left|\Delta_{\prec}(I)_{d, r}\right|=\left(\begin{array}{c}
H_{I}(d) \\
r
\end{array}\right),
$$

which is much smaller than the size of $\mathcal{F}_{\prec, d, r}^{p}$.

We come to one of our main results.

Theorem 4.9. Let $K$ be a field, let $\mathbb{X}$ be a finite subset of $\mathbb{P}^{s-1}$, and let $\prec$ be a monomial order. If $|\mathbb{X}| \geq 2$ and $\delta_{\mathbb{X}}(d, r)$ is the $r$-th generalized Hamming weight of $C_{\mathbb{X}}(d)$, then

$$
\mathrm{fp}_{I(\mathbb{X})}(d, r) \leq \delta_{\mathbb{X}}(d, r) \text { for } d \geq 1 \text { and } 1 \leq r \leq H_{I(\mathbb{X})}(d) .
$$

Proof. This follows from Theorem 4.5, Lemma 4.7, and Proposition 4.8

\section{Projective Nested Cartesian COdes}

In this section we introduce projective nested cartesian codes, a type of evaluation codes that generalize the classical projective Reed-Muller codes [22, 27, 33], and give a lower bound for the minimum distance of some of these codes.

Let $K=\mathbb{F}_{q}$ be a finite field, let $A_{1}, \ldots, A_{s}$ be a collection of subsets of $K$, and let

$$
\mathcal{X}=\left[A_{1} \times \cdots \times A_{s}\right]
$$

be the image of $A_{1} \times \cdots \times A_{s} \backslash\{0\}$ under the map $K^{s} \backslash\{0\} \rightarrow \mathbb{P}^{s-1}, x \rightarrow[x]$.

Definition 5.1. 4] The set $\mathcal{X}$ is called a projective nested cartesian set if

(i) $\{0,1\} \subset A_{i}$ for $i=1, \ldots, s$,

(ii) $a / b \in A_{j}$ for $1 \leq i<j \leq s, a \in A_{j}, 0 \neq b \in A_{i}$, and

(iii) $d_{1} \leq \cdots \leq d_{s}$, where $d_{i}=\left|A_{i}\right|$ for $i=1, \ldots, s$.

If $\mathcal{X}$ is a projective nested cartesian set and $C_{\mathcal{X}}(d)$ is its corresponding $d$-th projective ReedMuller-type code, we call $C_{\mathcal{X}}(d)$ a projective nested cartesian code.

The next conjecture is not true as will be shown in Example 6.1

Conjecture 5.2. (Carvalho, Lopez-Neumann, and López [4], [25, Conjecture 6.2]) Let $C_{\mathcal{X}}(d)$ be the $d$-th projective nested cartesian code on the set $\mathcal{X}=\left[A_{1} \times \cdots \times A_{s}\right]$ with $d_{i}=\left|A_{i}\right|$ for $i=1, \ldots, s$. Then its minimum distance is given by

$$
\delta_{\mathcal{X}}(d, 1)=\left\{\begin{array}{cl}
\left(d_{k+2}-\ell+1\right) d_{k+3} \cdots d_{s} & \text { if } d \leq \sum_{i=2}^{s}\left(d_{i}-1\right), \\
1 & \text { if } d \geq \sum_{i=2}^{s}\left(d_{i}-1\right)+1,
\end{array}\right.
$$

where $0 \leq k \leq s-2$ and $\ell$ are the unique integers such that $d=\sum_{i=2}^{k+1}\left(d_{i}-1\right)+\ell$ and $1 \leq \ell \leq d_{k+2}-1$. 
This conjecture is true in some special cases [4, Theorem 3.8], which includes the classical projective Reed-Muller codes [33]. The minimum distance of $C_{\mathcal{X}}(d)$ proposed in Conjecture 5.2 is in fact the minimum distance of a certain evaluation linear code [25, Corollary 6.9].

In what follows $\mathcal{X}=\left[A_{1} \times \cdots \times A_{s}\right]$ denotes a projective nested cartesian set, with $d_{i}=\left|A_{i}\right|$, and $C_{\mathcal{X}}(d)$ is its corresponding projective nested cartesian code. Throughout this section $\prec$ is the lexicographical order on $S$, with $t_{1} \prec \cdots \prec t_{s}$, and $\operatorname{in}_{\prec}(I(\mathcal{X}))$ is the initial ideal of $I(\mathcal{X})$.

Lemma 5.3. 25. Lemma 5.6] Let $1 \leq e_{1} \leq \cdots \leq e_{m}$ and $0 \leq b_{i} \leq e_{i}-1$ for $i=1, \ldots, m$ be integers. Then

$$
\prod_{i=1}^{m}\left(e_{i}-b_{i}\right) \geq\left(\sum_{i=1}^{k}\left(e_{i}-b_{i}\right)-(k-1)-\sum_{i=k+1}^{m} b_{i}\right) e_{k+1} \cdots e_{m}
$$

for $k=1, \ldots, m$, where $e_{k+1} \cdots e_{m}=1$ and $\sum_{i=k+1}^{m} b_{i}=0$ if $k=m$.

Lemma 5.4. Let $g$ be a homogeneous polynomial of $S$ of degree $1 \leq d \leq \sum_{i=1}^{s-1}\left(d_{i}-1\right)$ and let $[P]$ be a point of $\mathbb{P}^{s-1}$. If $g$ vanishes at all points of $\mathcal{X} \backslash\{[P]\}$, then $g(P)=0$.

Proof. Assume that $g(P) \neq 0$ and set $\mathcal{X}^{*}=A_{1} \times \cdots \times A_{s}$. One can write the point $[P]$ in standard form $[P]=\left(0, \ldots, 0, p_{k}, \ldots, p_{s}\right)$ with $p_{k}=1$. Notice that $V_{\mathcal{X}^{*}}(g)$, the zero set of $g$ in $\mathcal{X}^{*}$, has cardinality equal to $\left|\mathcal{X}^{*}\right|-\left(d_{k}-1\right)$. Setting $f_{i}=\prod_{\gamma \in A_{i}}\left(t_{i}-\gamma\right)$ for $1 \leq i \leq s$ and applying [23, Lemma 2.3], we get

$$
I\left(X^{*}\right)=\left(f_{1}, \ldots, f_{s}\right) \quad \text { and } \quad \operatorname{in}_{\prec}\left(I\left(\mathcal{X}^{*}\right)\right)=\left(t_{1}^{d_{1}}, \ldots, t_{s}^{d_{s}}\right) .
$$

Therefore, by the division algorithm, we can write

$$
g=h_{1} f_{1}+\cdots+h_{s} f_{s}+f,
$$

where $f(0)=0, \operatorname{deg}(f) \leq \operatorname{deg}(g)$, and $\operatorname{deg}_{t_{i}}(f) \leq d_{i}-1$ for all $i$. Thus

$$
\left|V_{\mathcal{X}^{*}}(f)\right|=\left|V_{\mathcal{X}^{*}}(g)\right|=d_{1} \cdots d_{s}-\left(d_{k}-1\right) .
$$

The initial term of $f$ has the form $t^{a}=t_{1}^{a_{1}} \cdots t_{s}^{a_{s}}$, where $a_{i} \leq d_{i}-1$ for all $i$. Hence

$$
\begin{aligned}
d_{1} \cdots d_{s}-\left(d_{k}-1\right) & =\left|V_{\mathcal{X}^{*}}(f)\right|=\operatorname{deg}\left(S /\left(I\left(\mathcal{X}^{*}\right), f\right)\right) \\
& \leq \operatorname{deg}\left(S /\left(\operatorname{in}_{\prec}\left(I\left(\mathcal{X}^{*}\right), \operatorname{in}_{\prec}(f)\right)=\operatorname{deg}\left(S /\left(t_{1}^{d_{1}}, \ldots, t_{s}^{d_{s}}, t^{a}\right)\right)\right)\right. \\
& =d_{1} \cdots d_{s}-\left(d_{1}-a_{1}\right) \cdots\left(d_{s}-a_{s}\right) .
\end{aligned}
$$

Thus $d_{k}-1 \geq\left(d_{1}-a_{1}\right) \cdots\left(d_{s}-a_{s}\right)$. Applying Lemma 5.3 with $m=k=s, e_{i}=d_{i}$, and $b_{i}=a_{i}$ for all $i$, we get

$$
\begin{aligned}
\left(d_{1}-a_{1}\right) \cdots\left(d_{s}-a_{s}\right) & \geq \sum_{i=1}^{s}\left(d_{i}-a_{i}\right)-(s-1) \\
& =\sum_{i=1}^{s-1}\left(d_{i}-1\right)-\sum_{i=1}^{s} a_{i}+d_{s} \geq d_{s} \geq d_{k} .
\end{aligned}
$$

Thus $\left(d_{1}-a_{1}\right) \cdots\left(d_{s}-a_{s}\right) \geq d_{k}$, a contradiction.

The next result gives a uniform upper bound for the number of zeros in a projective nested cartesian set for a family of homogeneous polynomials of fixed degree $d$, where $\mathrm{d}$ is within a certain range, and a corresponding lower bound for $\delta_{\mathcal{X}}(d, 1)$. 
Theorem 5.5. Let $f$ be a polynomial of degree $d$ that does not vanish at all points of $\mathcal{X}$. If $d=\sum_{i=2}^{s-1}\left(d_{i}-1\right)+\ell$ and $1 \leq \ell \leq d_{1}-1$, then

$$
\left|V_{\mathcal{X}}(f)\right| \leq \operatorname{deg}(S / I(\mathcal{X}))-\left(d_{1}-\ell+1\right)
$$

In particular $\delta_{\mathcal{X}}(d, 1) \geq d_{1}-\ell+1 \geq 2$.

Proof. We proceed by contradiction assuming that $\left|V_{\mathcal{X}}(f)\right| \geq|\mathcal{X}|-\left(d_{1}-\ell\right)$. If $n$ is the number of elements of $\mathcal{X} \backslash V_{\mathcal{X}}(f)$, then $n \leq d_{1}-\ell$. Let $\left[P_{1}\right], \ldots,\left[P_{n}\right]$ be the points of $\mathcal{X} \backslash V_{\mathcal{X}}(f)$. For each $1 \leq i \leq n-1$, pick $h_{i} \in S_{1}$ such that $h_{i}\left(P_{i}\right)=0$ and $h_{i}\left(P_{n}\right) \neq 0$. Setting $g=f h_{1} \cdots h_{n-1}$, one has

$$
\operatorname{deg}(g)=(n-1)+d=(n-1)+\sum_{i=2}^{s-1}\left(d_{i}-1\right)+\ell<d_{1}+\sum_{i=2}^{s-1}\left(d_{i}-1\right),
$$

$g\left(P_{n}\right) \neq 0$, and $g$ vanishes at all points of $\mathcal{X} \backslash\left\{\left[P_{n}\right]\right\}$. This contradicts Lemma 5.4 .

\section{EXAMPLES}

In this section we show some examples that illustrate how some of our results can be used in practice. In particular we give a counterexample to Conjecture 5.2 .

Example 6.1. Let $K$ be the field $\mathbb{F}_{4}$, let $\mathcal{X}$ be the projective nested cartesian set

$$
\mathcal{X}=\left[A_{1} \times A_{2} \times A_{3}\right] \subset \mathbb{P}^{2}
$$

where $A_{1}=\{0,1\}, A_{2}=\{0,1\}, A_{3}=\mathbb{F}_{4}$, and let $I=I(\mathcal{X})$ be the vanishing ideal of $\mathcal{X}$. The ideal $I$ is generated by $t_{1} t_{2}^{2}-t_{1}^{2} t_{2}, t_{1} t_{3}^{4}-t_{1}^{4} t_{3}, t_{2} t_{3}^{4}-t_{2}^{4} t_{3}, \operatorname{reg}(S / I)=5$, and $\operatorname{deg}(S / I)=13$. This follows from [4, Lemma 2.4]. Using Procedure [7.1, we obtain the following table with the basic parameters of $C_{\mathcal{X}}(d)$ :

\begin{tabular}{c|c|c|c|c|c|c}
$d$ & 1 & 2 & 3 & 4 & 5 & $\cdots$ \\
\hline$|\mathcal{X}|$ & 13 & 13 & 13 & 13 & 13 & $\cdots$ \\
\hline$H_{\mathcal{X}}(d)$ & 3 & 6 & 9 & 12 & 13 & $\cdots$ \\
\hline$\delta_{\mathcal{X}}(d, 1)$ & 8 & 4 & 3 & 1 & 1 & $\cdots$ \\
\hline $\operatorname{fp}_{I(\mathcal{X})}(d, 1)$ & 8 & 4 & 3 & 1 & 1 & $\cdots$
\end{tabular}

A direct way to see that $\delta_{\mathcal{X}}(4,1)=1$ is to observe that the polynomial $f=t_{3}\left(t_{3}^{3}-t_{2}^{3}-t_{1}^{3}+t_{1}^{2} t_{2}\right)$ vanishes at all points of $\mathcal{X} \backslash\left\{\left[e_{3}\right]\right\}$ and $f\left(e_{3}\right)=1$, where $e_{3}=(0,0,1)$. The ideal $I$ is Geil-Carvalho [26], that is, $\delta_{\mathcal{X}}(d, 1)=\mathrm{fp}_{I(\mathcal{X})}(d, 1)$ for $d \geq 1$. Using the notation of Conjecture 5.2, the values of $\delta_{\mathcal{X}}(d, 1)$ according to this conjecture are given by the following table:

\begin{tabular}{c|l|l|l|l|l|l}
$d$ & 1 & 2 & 3 & 4 & 5 & $\cdots$ \\
\hline$k$ & 0 & 1 & 1 & 1 & & \\
\hline$\ell$ & 1 & 1 & 2 & 3 & & \\
\hline$\delta_{\mathcal{X}}(d, 1)$ & 8 & 4 & 3 & 2 & 1 & $\cdots$
\end{tabular}

Thus the conjecture fails in degree $d=4$.

Example 6.2. Let $K$ be the field $\mathbb{F}_{4}$, let $\mathcal{X}$ be the projective nested cartesian set

$$
\mathcal{X}=\left[A_{1} \times A_{2} \times A_{3}\right] \subset \mathbb{P}^{2},
$$


where $A_{1}=\{0,1\}, A_{2}=\{0,1\}, A_{3}=\mathbb{F}_{4}$ and let $I=I(\mathcal{X})$ be the vanishing ideal of $\mathcal{X}$. Consider the following pairs of polynomials $F=\left\{f_{1}, f_{2}\right\}$ of degree $d$ :

Case $d=1$ : $\quad$ Case $d=2: \quad$ Case $d=3: \quad$ Case $d=4$ :

$f_{1}=t_{1}-t_{2}, \quad f_{1}=\left(t_{1}-t_{2}\right)\left(t_{1}-t_{3}\right), \quad f_{1}=\left(t_{1}-t_{2}\right)\left(t_{1}-t_{3}\right) t_{2}, \quad f_{1}=\left(t_{1}-t_{2}\right)\left(t_{1}-t_{3}\right) t_{2}^{2}$,

$f_{2}=t_{1}-t_{3} . \quad f_{2}=\left(t_{1}-t_{2}\right) t_{2} . \quad f_{2}=\left(t_{1}-t_{2}\right) t_{2}^{2} . \quad f_{2}=\left(t_{1}-t_{2}\right)\left(t_{2}-t_{3}\right) t_{2} t_{3}$.

If $V_{\mathcal{X}}(F)$ is the variety in $\mathcal{X}$ defined by $F=\left\{f_{1}, f_{2}\right\}$, using Procedure 7.2 we obtain:

Case $d=1:\left|V_{\mathcal{X}}(F)\right|=\operatorname{deg}(S /(I, F))=1, \quad$ Case $d=2:\left|V_{\mathcal{X}}(F)\right|=\operatorname{deg}(S /(I, F))=6$,

Case $d=3:\left|V_{\mathcal{X}}(F)\right|=\operatorname{deg}(S /(I, F))=9, \quad$ Case $d=4:\left|V_{\mathcal{X}}(F)\right|=\operatorname{deg}(S /(I, F))=10$.

Example 6.3. Let $\left(\operatorname{fp}_{I(\mathcal{X})}(d, r)\right)$ and $\left(\delta_{\mathcal{X}}(d, r)\right)$ be the footprint matrix and the weight matrix of the ideal $I(\mathcal{X})$ of Example 6.1. These matrices are of size $5 \times 13$ because the regularity and the degree of $S / I(\mathcal{X})$ are 5 and 13, respectively. Using Procedure 7.3 we obtain:

$$
\left(\operatorname{fp}_{I(\mathcal{X})}(d, r)\right)=\left[\begin{array}{ccccccccccccc}
8 & 12 & 13 & \infty & \infty & \infty & \infty & \infty & \infty & \infty & \infty & \infty & \infty \\
4 & 7 & 8 & 11 & 12 & 13 & \infty & \infty & \infty & \infty & \infty & \infty & \infty \\
3 & 4 & 6 & 7 & 8 & 10 & 11 & 12 & 13 & \infty & \infty & \infty & \infty \\
1 & 3 & 4 & 5 & 6 & 7 & 8 & 9 & 10 & 11 & 12 & 13 & \infty \\
1 & 2 & 3 & 4 & 5 & 6 & 7 & 8 & 9 & 10 & 11 & 12 & 13
\end{array}\right]
$$

If $r>H_{\mathcal{X}}(d)$, then $\mathcal{M}_{\prec, d, r}=\emptyset$ and the $(d, r)$-entry of this matrix is equal to $|\mathcal{X}|$, but in this case we prefer to write $\infty$ for computational reasons. Therefore, by Theorem 4.9, we obtain $\left(\mathrm{fp}_{I(\mathcal{X})}(d, r)\right) \leq\left(\delta_{\mathcal{X}}(d, r)\right)$. By Example 6.1 one has $\operatorname{fp}_{I(\mathcal{X})}(d, 1)=\delta_{\mathcal{X}}(d, 1)$ for $d \geq 1$ and by Example 6.2 it follows readily that $\operatorname{fp}_{I(\mathcal{X})}(d, 2)=\delta \mathcal{X}(d, 2)$ for $d \geq 1$. Using that the generalized footprint is a lower bound for the generalized Hamming weight we have verified that $\mathrm{fp}_{I(\mathcal{X})}(d, r)$ is equal to $\delta_{\mathcal{X}}(d, r)$ for all $d, r$.

Example 6.4. Let $\mathcal{X}=\mathbb{P}^{2}$ be the projective space over the field $\mathbb{F}_{2}$. The vanishing ideal $I=$ $I(\mathcal{X})$ is the ideal of $S=\mathbb{F}_{2}\left[t_{1}, t_{2}, t_{3}\right]$ generated by the binomials $t_{1} t_{2}^{2}-t_{1}^{2} t_{2}, t_{1} t_{3}^{2}-t_{1}^{2} t_{3}, t_{2}^{2} t_{3}-t_{2} t_{3}^{2}$ [27, Corollaire 2.1]. If $S$ has the GRevLex order $\prec$, adapting Procedure 7.1 we get

\begin{tabular}{c|l|l|l|l}
$d$ & 1 & 2 & 3 & $\cdots$ \\
\hline $\operatorname{deg}(S / I)$ & 7 & 7 & 7 & $\cdots$ \\
\hline$H_{I}(d)$ & 3 & 6 & 7 & $\cdots$ \\
\hline$\delta_{\mathcal{X}}(d, 1)$ & 4 & 2 & 1 & $\cdots$ \\
\hline $\operatorname{fp}_{I}(d, 1)$ & 4 & 1 & 1 & $\cdots$
\end{tabular}

\section{Procedures For Macaulay2}

In this section we present the procedures used to compute the examples of Section 6. Some of these procedures work for graded ideals and provide a tool to study generalized minimum distance functions.

Procedure 7.1. Computing the minimum distance and the footprint with Macaulay2 [17] using Theorem 4.5 and Proposition 4.8. The next procedure corresponds to Example 6.1.

$\mathrm{q}=4$

$\mathrm{G}=\mathrm{GF}(\mathrm{q}$, Variable $=>\mathrm{a})$

$\mathrm{S}=\mathrm{G}[\mathrm{t} 3, \mathrm{t} 2, \mathrm{t} 1$, MonomialOrder $=>\operatorname{Lex}]$

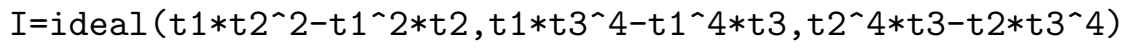

$M=$ coker gens $\mathrm{gb} I$, degree $M$, regularity $M$

init=ideal (leadTerm gens gb I) 
$\mathrm{H}=(\mathrm{d})->$ hilbertFunction $(\mathrm{d}, \mathrm{M}), \operatorname{apply}(1 \ldots \operatorname{regularity}(\mathrm{M}), \mathrm{H})$

$\mathrm{h}=(\mathrm{d})->$ degree $\mathrm{M}$ - max apply(apply(apply(apply(

toList $\left(\operatorname{set}\left(0, a, a^{\wedge} 2, a^{\wedge} 3\right)\right)^{\wedge} * *($ hilbertFunction $(d, M))-(\operatorname{set}\{0\})^{\wedge} * *($

hilbertFunction (d,M)), toList), $\mathrm{x}->$ basis $(\mathrm{d}, \mathrm{M}) *$ vector $\mathrm{x})$,

$z^{->i d e a l}(f l a t t e n$ entries $z)$ ),$x^{->}$if not quotient $(I, x)==I$ then degree

ideal $(I, x)$ else 0$)--h(d)$ is the minimum distance in degree $d$

apply $(1$. regularity $(M)-1, h)$

$f=(x)->$ if not quotient (init, $x$ )==init then degree ideal (init, $x$ ) else 0

$f p=(d)->$ degree $M-\max$ apply(flatten entries basis (d,M),f)

$--f p(d)$ is the footprint in degree $d$

apply (1..regularity (M),fp)

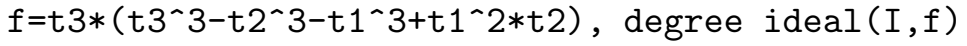

Procedure 7.2. Computing the number of solutions of a system of homogeneous polynomials over any given set of projective points over a finite field with Macaulay2 [17] using the degree formula of Lemma 4.5. The next procedure corresponds to Example 6.2.

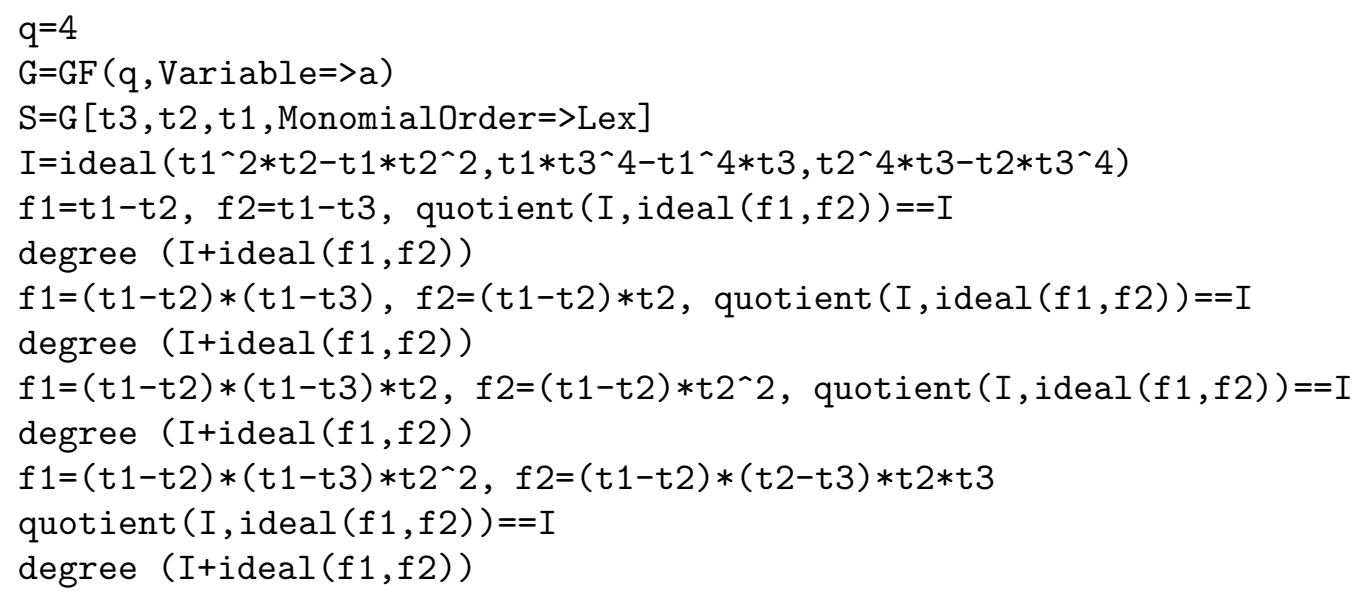

Procedure 7.3. Computing the footprint matrix with Macaulay2 [17]. This procedure corresponds to Example 6.3. It can be applied to any vanishing ideal $I$ to obtain the entries of the matrix $\left(\mathrm{fp}_{I}(d, r)\right)$ and is reasonably fast.

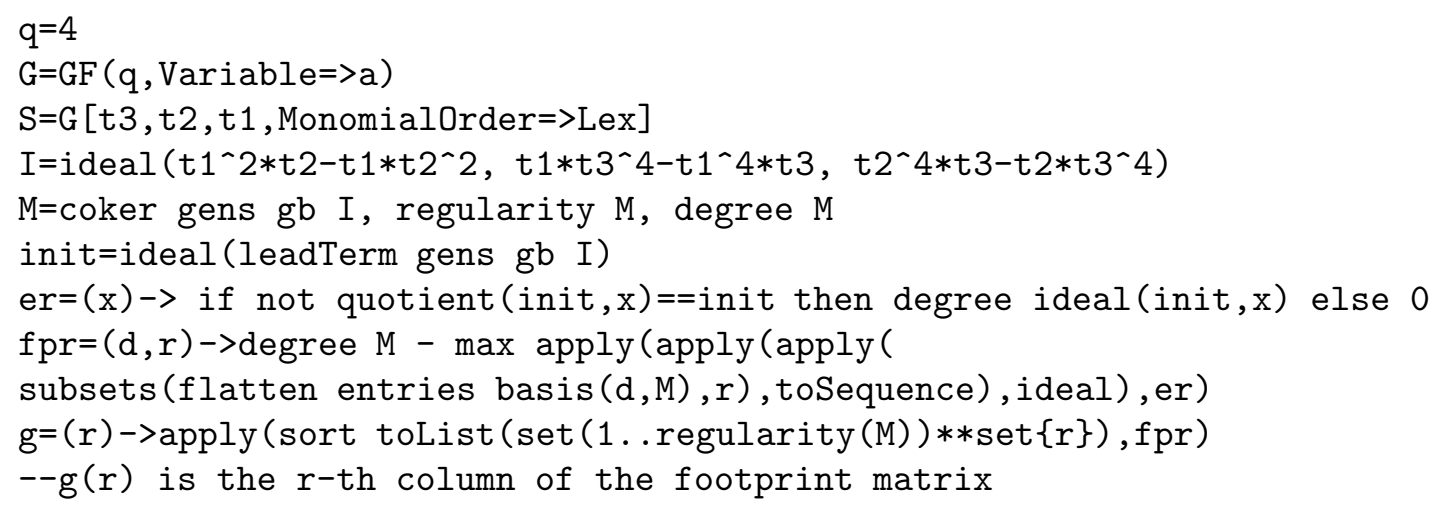




\section{An INTEGER INEQUALity}

For $a: a_{1}, \ldots, a_{m}$ and $b: b_{1}, \ldots, b_{m}$ sequences in $\mathbb{Z}^{+}=\{1,2, \ldots\}$ we define

$$
\pi(a, b):=\prod_{i=1}^{m} a_{i}+\prod_{i=1}^{m} b_{i}-\prod_{i=1}^{m} \min \left(a_{i}, b_{i}\right) .
$$

Lemma 8.1. Let $a_{1}, a_{2}, b_{1}, b_{2} \in \mathbb{Z}^{+}$. Set $a_{1}^{\prime}=\min \left(a_{1}, a_{2}\right)$ and $a_{2}^{\prime}=\max \left(a_{1}, a_{2}\right)$. Then

$$
\min \left(a_{1}, b_{1}\right) \min \left(a_{2}, b_{2}\right) \leq \min \left(a_{1}^{\prime}, b_{1}^{\prime}\right) \min \left(a_{2}^{\prime}, b_{2}^{\prime}\right) .
$$

Proof. It is an easy case-by-case verification of 4 ! possible cases.

Lemma 8.2. Let $a: a_{1}, \ldots, a_{m}$ and $b: b_{1}, \ldots, b_{m}$ be sequences in $\mathbb{Z}^{+}$. Suppose:

(i) $r<s, a_{r}>a_{s}$. Set $a_{r}^{\prime}=a_{s}, a_{s}^{\prime}=a_{r}, a_{i}^{\prime}=a_{i}$ for $i \neq r, s$; and $b_{r}^{\prime}=\min \left(b_{r}, b_{s}\right)$, $b_{s}^{\prime}=\max \left(b_{r}, b_{s}\right), b_{i}^{\prime}=b_{i}$ for $i \neq r, s$. Then $\pi(a, b) \geq \pi\left(a^{\prime}, b^{\prime}\right)$.

(ii) $r<s, b_{r}=a_{r} \leq a_{s}<b_{s}$. Set $a_{r}^{\prime}=a_{r}-1, a_{s}^{\prime}=a_{s}+1, a_{i}^{\prime}=a_{i}$ for $i \neq r$, $s$. Then $\pi(a, b) \geq \pi\left(a^{\prime}, b\right)$.

(iii) $r<s, b_{r}<a_{r} \leq a_{s}$. Set $a_{r}^{\prime}=a_{r}-1, a_{s}^{\prime}=a_{s}+1, a_{i}^{\prime}=a_{i}$ for $i \neq r, s$. Then $\pi(a, b) \geq \pi\left(a^{\prime}, b\right)$.

(iv) $r<s, a_{r}<a_{s}, b_{r}=a_{s}, b_{s}=a_{r}, b_{i}=a_{i}$ for $i \neq r, s, h:=a_{s}-a_{r} \geq 2$. Set $b_{r}^{\prime}=a_{r}+1$, $b_{s}^{\prime}=a_{s}-1, b_{i}^{\prime}=a_{i}$ for $i \neq r, s$. Then $\pi(a, b) \geq \pi\left(a, b^{\prime}\right)$.

Proof. We verify all cases by direct substitution of $a^{\prime}$ and $b^{\prime}$ into $\pi(a, b)$.

$$
\begin{aligned}
& \text { (i) } \pi(a, b)-\pi\left(a^{\prime}, b^{\prime}\right)=\prod a_{i}+\prod b_{i}-\prod a_{i}^{\prime}-\prod b_{i}^{\prime}+\prod \min \left(a_{i}^{\prime}, b_{i}^{\prime}\right)-\prod \min \left(a_{i}, b_{i}\right) \\
& =\left(\min \left(a_{r}^{\prime}, b_{r}^{\prime}\right) \min \left(a_{s}^{\prime}, b_{s}^{\prime}\right)-\min \left(a_{r}, b_{r}\right) \min \left(a_{s}, b_{s}\right)\right) \prod_{i \neq r, s} \min \left(a_{i}, b_{i}\right) \geq 0 . \quad \text { (Lemma 8.1) } \\
& \text { (ii) } \pi(a, b)-\pi\left(a^{\prime}, b\right)=\prod a_{i}-\prod a_{i}^{\prime}+\prod \min \left(a_{i}^{\prime}, b_{i}\right)-\prod \min \left(a_{i}, b_{i}\right) \\
& =\left(a_{r} a_{s}-\left(a_{r}-1\right)\left(a_{s}+1\right)\right) \prod_{i \neq r, s} a_{i} \\
& +\left(\min \left(a_{r}^{\prime}, b_{r}\right) \min \left(a_{s}^{\prime}, b_{s}\right)-\min \left(a_{r}, b_{r}\right) \min \left(a_{s}, b_{s}\right)\right) \prod_{i \neq r, s} \min \left(a_{i}, b_{i}\right) \\
& =\left(a_{s}-a_{r}+1\right) \prod_{i \neq r, s} a_{i}+\left(\left(a_{r}-1\right)\left(a_{s}+1\right)-a_{r} a_{s}\right) \prod_{i \neq r, s} \min \left(a_{i}, b_{i}\right) \\
& =\left(a_{s}-a_{r}+1\right)\left(\prod_{i \neq r, s} a_{i}-\prod_{i \neq r, s} \min \left(a_{i}, b_{i}\right)\right) \geq 0 \text {. } \\
& \text { (iii) } \pi(a, b)-\pi\left(a^{\prime}, b\right)=\prod a_{i}-\prod a_{i}^{\prime}+\prod \min \left(a_{i}^{\prime}, b_{i}\right)-\prod \min \left(a_{i}, b_{i}\right) \\
& =\left(a_{r} a_{s}-\left(a_{r}-1\right)\left(a_{s}+1\right)\right) \prod_{i \neq r, s} a_{i} \\
& +\left(\min \left(a_{r}^{\prime}, b_{r}\right) \min \left(a_{s}^{\prime}, b_{s}\right)-\min \left(a_{r}, b_{r}\right) \min \left(a_{s}, b_{s}\right)\right) \prod_{i \neq r, s} \min \left(a_{i}, b_{i}\right) \\
& =\left(a_{s}-a_{r}+1\right) \prod_{i \neq r, s} a_{i}+b_{r}\left(\min \left(a_{s}+1, b_{s}\right)-\min \left(a_{s}, b_{s}\right)\right) \prod_{i \neq r, s} \min \left(a_{i}, b_{i}\right) \geq 0 .
\end{aligned}
$$


For the last inequality note that $\min \left(a_{s}+1, b_{s}\right)-\min \left(a_{s}, b_{s}\right)=0$ or 1 .

$$
\text { (iv) } \begin{aligned}
\pi(a, b)-\pi\left(a, b^{\prime}\right) & =\prod b_{i}-\prod b_{i}^{\prime}+\prod \min \left(a_{i}, b_{i}^{\prime}\right)-\prod \min \left(a_{i}, b_{i}\right) \\
& =\left(a_{r} a_{s}-\left(a_{r}+1\right)\left(a_{s}-1\right)+a_{r}\left(a_{s}-1\right)-a_{r}^{2}\right) \prod_{i \neq r, s} a_{i} \\
& =\left(a_{r}-1\right)(h-1) \prod_{i \neq r, s} a_{i} \geq 0 . \quad \square
\end{aligned}
$$

Lemma 8.3. If $a_{1}, \ldots, a_{r}$ are positive integers, then $a_{1} \cdots a_{r} \geq\left(a_{1}+\cdots+a_{r}\right)-(r-1)$.

Proof. It follows by induction on $r$.

Lemma 8.4. Let $1 \leq e_{1} \leq \cdots \leq e_{m}$ and $1 \leq a_{i}, b_{i} \leq e_{i}$, for $i=1, \ldots, m$ be integers. Suppose $a_{i}=b_{i}=1$ for $i<r, a_{i}=b_{i}=e_{i}$ for $i>r+1:=s, 1 \leq a_{i}, b_{i} \leq e_{i}$ for $i=r, s$, with $a_{r}+a_{s}=b_{r}+b_{s}$ and $\left(a_{r}, a_{s}\right) \neq\left(b_{r}, b_{s}\right)$. If $b_{r} \leq a_{s}$ and $b_{s}=a_{s}-1$, then

$$
\pi(a, b) \geq\left(\sum_{i=1}^{m} a_{i}-\sum_{i=k+1}^{m} e_{i}-(k-2)\right) e_{k+1} \cdots e_{m}-e_{k+2} \cdots e_{m}
$$

for $i=1, \ldots, m-1$, where $e_{k+2} \cdots e_{m}=1$ when $k=m-1$.

Proof. Set $\sigma=\sum_{i=1}^{m} a_{i}-\sum_{i=k+1}^{m} e_{i}-(k-2)$. Since $b_{s}\left(b_{r}-a_{r}\right)=a_{s}-1$, one has the equality

$$
\pi(a, b)=\left(a_{r} a_{s}+b_{r} b_{s}-a_{r} b_{s}\right) \prod_{i=r+2}^{m} e_{i}=\left(a_{r} a_{s}+a_{s}-1\right) \prod_{i=r+2}^{m} e_{i} .
$$

Case $k+1<r$ : The integer $\sigma$ can be rewritten as

$$
\sigma=k+\left(1-e_{k+1}\right)+\cdots+\left(1-e_{r-1}\right)+\left(a_{r}-e_{r}\right)+\left(a_{s}-e_{s}\right)-(k-2) .
$$

Since $a_{r}<b_{r} \leq e_{r}$, it holds that $a_{r}-e_{r} \leq-1$, and hence $\sigma \leq 1$. If $\sigma \leq 0$, Eq. (8.1) trivially follows (because the left hand side is positive and the right hand side would be negative). So we may assume $\sigma=1$. This assumption implies that $e_{k+1}=1$ because $a_{r}<b_{r} \leq e_{r}$. Then the right hand side of Eq. (8.1) is

$$
(\sigma) e_{k+1} \cdots e_{m}-e_{k+2} \cdots e_{m}=\left(e_{k+1}-1\right) e_{k+2} \cdots e_{r}=0 .
$$

Case $k+1=r$ : The integer $\sigma$ can be rewritten as

$$
\sigma=k+\left(a_{r}-e_{r}\right)+\left(a_{s}-e_{s}\right)-(k-2) .
$$

By the same reason as above, we may assume $\sigma=1$. This assumption implies $a_{r}=e_{r}-1$ and $a_{s}=e_{s}$. Then, by Eq. (8.2), we obtain that Eq. (8.1) is equivalent to

$$
\left(e_{r} e_{s}-1\right) \prod_{i=r+2}^{m} e_{i} \geq(\sigma) e_{r} \cdots e_{m}-e_{r+1} \cdots e_{m}
$$

which reduces to $e_{r} e_{s}-1 \geq(1) e_{r} e_{s}-e_{s}$, or equivalently, $e_{s} \geq 1$.

Case $k+1=r+1$ : We can rewrite $\sigma$ as

$$
\sigma=(k-1)+a_{r}+\left(a_{s}-e_{s}\right)-(k-2)=a_{r}+\left(a_{s}-e_{s}\right)+1 .
$$


Then, using Eq. (8.2), we obtain that Eq. (8.1) is equivalent to

$$
\left(a_{r} a_{s}+a_{s}-1\right) \prod_{i=r+2}^{m} e_{i} \geq(\sigma) e_{r+1} \cdots e_{m}-e_{r+2} \cdots e_{m},
$$

which reduces to $a_{r} a_{s}+a_{s} \geq\left(a_{r}+a_{s}-e_{s}+1\right) e_{s}$, or equivalently,

$$
\left(e_{s}-a_{s}\right)\left(e_{s}-a_{r}-1\right) \geq 0 \text {. }
$$

Case $k+1>r+1$ : One can rewrite $\sigma$ as

$$
\sigma=(r-1)+a_{r}+\cdots+a_{k}-(k-2) .
$$

Then, using Eq. (8.2), we obtain that Eq. (8.1) reduces to

$$
\left(a_{r} a_{s}+a_{s}-1\right) e_{r+2} \cdots e_{k+1} \geq(\sigma) e_{k+1}-1 .
$$

But, as $a_{s} \geq 2$, using Lemma 8.3 , we get

$$
\begin{aligned}
\left(a_{r} a_{s}+a_{s}-1\right) e_{r+2} \cdots e_{k} & \geq\left(a_{r} a_{s}+a_{s}-1\right)+e_{r+2}+\cdots+e_{k}-(k-r-1) \\
& \geq\left(a_{r}+a_{s}\right)+a_{r+2}+\cdots+a_{k}+r-k+1=\sigma .
\end{aligned}
$$

So, multiplying by $e_{k+1}$, the required inequality follows.

Theorem 8.5. Let $d \geq 1$ and $1 \leq e_{1} \leq \cdots \leq e_{m}$ be integers. Suppose $1 \leq a_{i} \leq e_{i}$ and $1 \leq b_{i} \leq e_{i}$, for $i=1, \ldots, m$, are integers such that $d=\sum_{i=1}^{m} a_{i}=\sum_{i=1}^{m} b_{i}$ and $a \neq b$. Then

$$
\pi(a, b) \geq\left(\sum_{i=1}^{m} a_{i}-\sum_{i=k+1}^{m} e_{i}-(k-2)\right) e_{k+1} \cdots e_{m}-e_{k+2} \cdots e_{m}
$$

for $k=1, \ldots, m-1$, where $e_{k+2} \cdots e_{m}=1$ when $k=m-1$.

Proof. Apply to $(a, b)$ any of the four "operations" described in Lemma 8.2, and let $\left(a^{\prime}, b^{\prime}\right)$ be the new obtained pair. These operations should be applied in such a way that $1 \leq a_{i}^{\prime}, b_{i}^{\prime} \leq e_{i}$ for $i=1, \ldots, m$ and $a^{\prime} \neq b^{\prime}$; this is called a valid operation. One can order the set of all pairs $(a, b)$ that satisfy the hypothesis of the proposition using the GRevLex order defined by $(a, b) \succ\left(a^{\prime}, b^{\prime}\right)$ if and only if the last non-zero entry of $(a, b)-\left(a^{\prime}, b^{\prime}\right)$ is negative. Note that by construction $d=\sum a_{i}^{\prime}=\sum b_{i}^{\prime}=\sum a_{i}$. Repeat this step as many times as possible (which is a finite number because the result $\left(a^{\prime}, b^{\prime}\right)$ of any valid operation applied to $(a, b)$ satisfies $\left.(a, b) \succ\left(a^{\prime}, b^{\prime}\right)\right)$. Permitting an abuse of notation, let $a$ and $b$ be the resulting sequences at the end of that process. We will show that these $a$ and $b$ satisfy the hypothesis of Lemma 8.4 .

Set $r=\min \left(i: a_{i} \neq b_{i}\right)$. By symmetry we may assume $a_{r}<b_{r}$. Pick the first $s>r$ such that $a_{s}>b_{s}$ (the case $a_{r}>b_{r}$ and $a_{s}<b_{s}$ can be shown similarly).

Claim (a): For $p<r, a_{p}=1$. Assume $a_{p}>1$. If $a_{p}>a_{r}$, we can apply Lemma $8.2(i)[p, r]$, which is assumed not possible; (this last notation means that we are applying Lemma $8.2(i)$ with the indexes $p$ and $r$ ). Otherwise apply Lemma 8.2 $(i i)[p, r]$. So $a_{p}=b_{p}=1$ for $p<r$.

Claim (b): $s=r+1$. Suppose $r<p<s$. To obtain a contradiction it suffices to show that we can apply a valid operation to $a, b$. By the choice of $s, a_{p} \leq b_{p}$. If $b_{r}>b_{p}$, we can apply Lemma $8.2(i)[r, p]$. If $b_{p}>b_{s}$, we can apply Lemma $8.2(i)[p, s]$. Hence $b_{r} \leq b_{p} \leq b_{s}$. Notice that $b_{p} \geq 2$ because $a_{r}<b_{r} \leq b_{p}$. If $a_{p}=b_{p}$, we can apply Lemma $8.2(i i)[p, s]$. If $a_{p}<b_{p}$, we can apply Lemma $8.2($ iii $)[p, s]$ because $a_{p}<b_{p} \leq b_{s}<a_{s}$.

Claim (c): For $p>s, a_{p}=b_{p}=e_{p}$. If $b_{p}<a_{p}$, applying Claim (b) to $r$ and $p$ we get a contradiction. Thus we may assume $b_{p} \geq a_{p}$. It suffices to show that $a_{p}=e_{p}$. If $a_{s}>a_{p}$, then by Lemma $\underline{8.2}(i)[s, p]$ one can apply a valid operation to $a, b$, a contradiction. Thus $a_{s} \leq a_{p}$. If 
$a_{p}<e_{p}$, then $b_{s}<a_{s} \leq a_{p}<e_{p}$, and by Lemma $8.2(i i i)[s, p]$ we can apply a valid operation to $a, b$, a contradiction. Hence $a_{p}=e_{p}$.

Claim (d): $b_{r} \leq a_{s}$ and $b_{s}=a_{s}-1$. By the previous claims one has the equalities $s=r+1$ and $a_{r}+a_{s}=b_{r}+b_{s}$. If $a_{s}<b_{r}$. Then $b_{s}<a_{s}<b_{r}$, and by Lemma $8.2(i)[r, s]$ we can apply a valid operation to $a, b$, a contradiction. Hence $a_{s} \geq b_{r}$. Suppose $a_{s}=b_{r}$, then $a_{r}=b_{s}$. If $a_{s}-a_{r} \geq 2$, by Lemma $8.2(i v)[r, s]$ we can apply a valid operation to $a, b$, a contradiction. Hence, in this case, $a_{s}-b_{s}=a_{s}-a_{r}=1$. Suppose $a_{s}>b_{r}$. If $b_{r}>b_{s}$, then $a_{s}>b_{r}>b_{s}$, and we can use Lemma $8.2(i)[r, s]$ to apply a valid operation to $a, b$, a contradiction. Hence $b_{r} \leq b_{s}$. If $a_{s}-b_{s}=b_{r}-a_{r} \geq 2$, then $a_{r}<b_{r} \leq b_{s}<a_{s}$, and by Lemma $8.2(i i i)[r, s]$ we can apply a valid operation to $a, b$, a contradiction. So, in this other case, also $a_{s}-b_{s}=1$. In conclusion, we have that $b_{r} \leq a_{s}$ and $b_{s}=a_{s}-1$, as claimed.

From Claims (a)-(d), we obtain that $a, b$ satisfy the hypothesis of Lemma 8.4. Hence the required inequality follows from Lemmas 8.2 and 8.4 .

For $\alpha: \alpha_{1}, \ldots, \alpha_{n}$ and $\beta: \beta_{1}, \ldots, \beta_{n}$ sequences in $\mathbb{Z}^{+}$we define

$$
P(\alpha, \beta)=\prod_{i=1}^{n}\left(d_{i}-\alpha_{i}\right)+\prod_{i=1}^{n}\left(d_{i}-\beta_{i}\right)-\prod_{i=1}^{n} \min \left\{d_{i}-\alpha_{i}, d_{i}-\beta_{i}\right\} .
$$

Lemma 8.6. Let $1 \leq d_{1} \leq \cdots \leq d_{n}, 0 \leq \alpha_{i}, \beta_{i} \leq d_{i}-1$ for $i=1, \ldots, n, n \geq 2$, be integers such that $\sum_{i=1}^{n} \alpha_{i}=\sum_{i=1}^{n} \beta_{i}$ and $\left(\alpha_{1}, \ldots, \alpha_{n}\right) \neq\left(\beta_{1}, \ldots, \beta_{n}\right)$. Then

$$
P(\alpha, \beta) \geq\left(\sum_{i=1}^{k+1}\left(d_{i}-\alpha_{i}\right)-(k-1)-\sum_{i=k+2}^{n} \alpha_{i}\right) d_{k+2} \cdots d_{n}-d_{k+3} \cdots d_{n}
$$

for $k=0, \ldots, n-2$, where $d_{k+3} \cdots d_{n}=1$ if $k=n-2$.

Proof. Making the substitutions $m=n, k=k-1, d_{i}-\alpha_{i}=a_{i}, d_{i}-\beta_{i}=b_{i}$, and $d_{i}=e_{i}$, the inequality follows at once from Theorem 8.5 .

\section{Second generalized Hamming Weight}

Let $A_{1}, \ldots, A_{s-1}$ be subsets of $\mathbb{F}_{q}$ and let $\mathbb{X}:=\left[A_{1} \times \cdots \times A_{s-1} \times\{1\}\right] \subset \mathbb{P}^{s-1}$ be a projective cartesian set, where $d_{i}=\left|A_{i}\right|$ for all $i=1, \ldots, s-1$ and $2 \leq d_{1} \leq \cdots \leq d_{s-1}$. The Reed-Mullertype code $C_{\mathbb{X}}(d)$ is called an affine cartesian code [23]. If $\mathbb{X}^{*}=A_{1} \times \cdots \times A_{s-1}$, then $C_{\mathbb{X}}(d)=$ $C_{\mathbb{X}^{*}}(d)$ [23]. Assume $d=\sum_{i=1}^{k}\left(d_{i}-1\right)+\ell$, where $k, \ell$ are integers such that $0 \leq k \leq s-2$ and $1 \leq \ell \leq d_{k+1}-1$.

Lemma 9.1. We can find two linearly independent polynomials $F$ and $G \in S_{\leq d}$ such that

$$
\left|V_{\mathbb{X}^{*}}(F) \cap V_{\mathbb{X}^{*}}(G)\right|= \begin{cases}d_{1} \cdots d_{s-1}-\left(d_{k+1}-\ell+1\right) d_{k+2} \cdots d_{s-1}+d_{k+3} \cdots d_{s-1} & \text { if } k<s-3, \\ d_{1} \cdots d_{s-1}-\left(d_{k+1}-\ell+1\right) d_{k+2} \cdots d_{s-1}+1 & \text { if } k=s-3, \\ d_{1} \cdots d_{s-1}-d_{s-1}+\ell-1 & \text { if } k=s-2 .\end{cases}
$$

Proof. Case (I): $k \leq s-3$. Similarly to [23] we take $A_{i}=\left\{\beta_{i, 1}, \ldots, \beta_{i, d_{i}}\right\}$, for $i=1, \ldots, s-1$. Also, for $i=1, \ldots, k$, let

$$
\begin{aligned}
& f_{i}:=\left(\beta_{i, 1}-t_{i}\right)\left(\beta_{i, 2}-t_{i}\right) \cdots\left(\beta_{i, d_{i-1}}-t_{i}\right), \\
& g:=\left(\beta_{k+1,1}-t_{k+1}\right)\left(\beta_{k+1,2}-t_{k+1}\right) \cdots\left(\beta_{k+1, \ell-1}-t_{k+1}\right) .
\end{aligned}
$$


Setting $h_{1}:=\beta_{k+1, \ell}-t_{k+1}$ and $h_{2}:=\beta_{k+2, \ell}-t_{k+2}$. We define $F:=f_{1} \cdots f_{k} \cdot g \cdot h_{1}$ and $G:=f_{1} \cdots f_{k} \cdot g \cdot h_{2}$. Notice that $\operatorname{deg} F=\operatorname{deg} G=\sum_{i=1}^{k}\left(d_{i}-1\right)+\ell=d$ and that they are linearly independent over $\mathbb{F}_{q}$. Let

$$
\begin{aligned}
& V_{1}:=\left(A_{1} \times \cdots \times A_{s-1}\right) \backslash\left(V_{\mathbb{X}^{*}}(F) \cap V_{\mathbb{X}^{*}}(G)\right), \\
& V_{2}:=\left\{\beta_{1, d_{1}}\right\} \times \cdots \times\left\{\beta_{k, d_{k}}\right\} \times\left\{\beta_{k+1, i}\right\}_{i=\ell}^{d_{k+1}} \times A_{k+2} \times \cdots \times A_{s-1} .
\end{aligned}
$$

It is easy to see that $V_{1} \subset V_{2}$ and $\left(V_{2} \backslash V_{1}\right) \cap\left(V_{\mathbb{X}^{*}}(F) \cap V_{\mathbb{X}^{*}}(G)\right)=V_{3}$, where

$$
V_{3}= \begin{cases}\left\{\beta_{1, d_{1}}\right\} \times \cdots \times\left\{\beta_{k, d_{k}}\right\} \times\left\{\beta_{k+1, \ell}\right\} \times\left\{\beta_{k+2, \ell}\right\} \times A_{k+3} \times \cdots \times A_{s-1} & \text { if } k<s-3, \\ \left\{\beta_{1, d_{1}}\right\} \times \cdots \times\left\{\beta_{k, d_{k}}\right\} \times\left\{\beta_{k+1, \ell}\right\} \times\left\{\beta_{k+2, \ell}\right\} & \text { if } k=s-3 .\end{cases}
$$

Therefore

$$
\left|V_{1}\right|=\left|V_{2}\right|-\left|V_{3}\right|= \begin{cases}\left(d_{k+1}-\ell+1\right) d_{k+2} \cdots d_{s-1}-d_{k+3} \cdots d_{s-1} & \text { if } \quad k<s-3 \\ \left(d_{k+1}-\ell+1\right) d_{k+2} \cdots d_{s-1}-1 & \text { if } \quad k=s-3\end{cases}
$$

and the claim follows because $\left|V_{\mathbb{X}^{*}}(F) \cap V_{\mathbb{X}^{*}}(G)\right|=d_{1} \cdots d_{s-1}-\left|V_{1}\right|$.

Case (II): $k=s-2$. As $\ell \leq d_{k+1}-1$ then $\ell+1 \leq d_{k+1}$. Let $h_{3}:=\beta_{k+1, \ell+1}-t_{k+1}$, and $F, f_{i}, g, h_{1}$ as in Case (I). Let $G^{\prime}:=f_{1} \cdots f_{k} \cdot g \cdot h_{3}$. If

$$
\begin{aligned}
& V_{1}^{\prime}:=\left(A_{1} \times \cdots \times A_{s-1}\right) \backslash\left(V_{\mathbb{X}^{*}}(F) \cap V_{\mathbb{X}^{*}}\left(G^{\prime}\right)\right), \\
& V_{2}^{\prime}:=\left\{\beta_{1, d_{1}}\right\} \times \cdots \times\left\{\beta_{k, d_{k}}\right\} \times\left\{\beta_{k+1, i}\right\}_{i=\ell}^{d_{k+1}},
\end{aligned}
$$

then (because $h_{1}$ and $h_{3}$ do not have common zeros) $V_{1}^{\prime}=V_{2}^{\prime}$ and thus

$$
\left|V_{1}^{\prime}\right|=d_{k+1}-\ell+1=d_{s-1}-\ell+1 .
$$

The result follows because $\left|V_{\mathbb{X}^{*}}(F) \cap V_{\mathbb{X}^{*}}\left(G^{\prime}\right)\right|=d_{1} \cdots d_{s-1}-\left|V_{1}^{\prime}\right|$.

Lemma 9.2. [26, Lemma 3.3] Let $L \subset S$ be the ideal $\left(t_{1}^{d_{1}}, \ldots, t_{s-1}^{d_{s-1}}\right)$, where $d_{1}, \ldots, d_{s-1}$ are in $\mathbb{N}_{+}$. If $t^{a}=t_{1}^{a_{1}} \cdots t_{s}^{a_{s}}, a_{j} \geq 1$ for some $1 \leq j \leq s-1$, and $a_{i} \leq d_{i}-1$ for $i \leq s-1$, then

$$
\operatorname{deg}\left(S /\left(L, t^{a}\right)\right)=\operatorname{deg}\left(S /\left(L, t_{1}^{a_{1}} \cdots t_{s-1}^{a_{s-1}}\right)\right)=d_{1} \cdots d_{s-1}-\prod_{i=1}^{s-1}\left(d_{i}-a_{i}\right) .
$$

We come to one of our applications to coding theory.

Theorem 9.3. Let $A_{i}, i=1, \ldots, s-1$, be subsets of $\mathbb{F}_{q}$ and let $\mathbb{X} \subset \mathbb{P}^{s-1}$ be the projective cartesian set given by $\mathbb{X}=\left[A_{1} \times \cdots \times A_{s-1} \times\{1\}\right]$. If $d_{i}=\left|A_{i}\right|$ for $i=1, \ldots, s-1$ and $2 \leq d_{1} \leq \cdots \leq d_{s-1}$, then

$$
\delta_{\mathbb{X}}(d, 2)=\left\{\begin{array}{cl}
\left(d_{k+1}-\ell+1\right) d_{k+2} \cdots d_{s-1}-d_{k+3} \cdots d_{s-1} & \text { if } k<s-3, \\
\left(d_{k+1}-\ell+1\right) d_{k+2} \cdots d_{s-1}-1 & \text { if } k=s-3, \\
d_{s-1}-\ell+1 & \text { if } k=s-2, \\
2 & \text { if } d \geq \sum_{i=1}^{s-1}\left(d_{i}-1\right),
\end{array}\right.
$$

where $0 \leq k \leq s-2$ and $\ell$ are integers such that $d=\sum_{i=1}^{k}\left(d_{i}-1\right)+\ell$ and $1 \leq \ell \leq d_{k+1}-1$.

Proof. We set $n=s-1, I=I(\mathbb{X})$, and $L=\left(t_{1}^{d_{1}}, \ldots, t_{n}^{d_{n}}\right)$. By [37, Theorem 1, Corollary 1], we get $\delta_{\mathbb{X}}(d, 2)=2$ for $d \geq \sum_{i=1}^{s-1}\left(d_{i}-1\right)$. Thus we may assume $d<\sum_{i=1}^{s-1}\left(d_{i}-1\right)$. First we show the inequality " $\geq$ ". Let $\prec$ be a graded monomial order with $t_{1} \succ \cdots \succ t_{s}$. The initial ideal $\operatorname{in}_{\prec}(I)$ of $I$ is equal to $L=\left(t_{1}^{d_{1}}, \ldots, t_{n}^{d_{n}}\right)$; see [23. Let $F=\left\{t^{a}, t^{b}\right\}$ be an element of $\mathcal{M}_{\prec, d, 2}$, that is, $t^{a}=t_{1}^{a_{1}} \cdots t_{s}^{a_{s}}, t^{b}=t_{1}^{b_{1}} \cdots t_{s}^{b_{s}}, d=\sum_{i=1}^{s} a_{i}=\sum_{i=1}^{s} b_{i}, a \neq b, a_{i} \leq d_{i}-1$ and $b_{i} \leq d_{i}-1$ 
for $i=1, \ldots, n$, and $(L:(F)) \neq L$. In particular, from the last condition it follows readily that $a_{i} \neq 0$ and $b_{j} \neq 0$ for some $1 \leq i, j \leq n$. There are exact sequences of graded $S$-modules

$$
\begin{aligned}
0 \rightarrow & \left(S /\left(\left(L, t^{a}\right): t^{b}\right)\right)[-|b|] \stackrel{t^{b}}{\rightarrow} S /\left(L, t^{a}\right) \rightarrow S /\left(L, t^{a}, t^{b}\right) \rightarrow 0, \\
& 0 \rightarrow\left(S /\left(\left(L, t^{b}\right): t^{a}\right)\right)[-|a|] \stackrel{t^{a}}{\rightarrow} S /\left(L, t^{b}\right) \rightarrow S /\left(L, t^{a}, t^{b}\right) \rightarrow 0,
\end{aligned}
$$

where $|a|=\sum_{i=1}^{s} a_{i}$. From the equalities

$$
\begin{aligned}
& \left(\left(L, t^{a}\right): t^{b}\right)=\left(L: t^{b}\right)+\left(t^{a}: t^{b}\right)=\left(t_{1}^{d_{1}-b_{1}}, \ldots, t_{n}^{d_{n}-b_{n}}, \prod_{i=1}^{s} t_{i}^{\max \left\{a_{i}, b_{i}\right\}-b_{i}}\right), \\
& \left(\left(L, t^{b}\right): t^{a}\right)=\left(L: t^{a}\right)+\left(t^{b}: t^{a}\right)=\left(t_{1}^{d_{1}-a_{1}}, \ldots, t_{n}^{d_{n}-a_{n}}, \prod_{i=1}^{s} t_{i}^{\max \left\{a_{i}, b_{i}\right\}-a_{i}}\right),
\end{aligned}
$$

it follows that either $\left(\left(L, t^{a}\right): t^{b}\right)$ or $\left(\left(L, t^{b}\right): t^{a}\right)$ is contained in $\left(t_{1}, \ldots, t_{n}\right)$. Hence at least one of these ideals has height $n$. Therefore, setting

$$
P(a, b)=\prod_{i=1}^{n}\left(d_{i}-a_{i}\right)+\prod_{i=1}^{n}\left(d_{i}-b_{i}\right)-\prod_{i=1}^{n} \min \left\{d_{i}-a_{i}, d_{i}-b_{i}\right\}
$$

and using Lemma 9.2 it is not hard to see that the degree of $S /\left(L, t^{a}, t^{b}\right)$ is

$$
\operatorname{deg}\left(S /\left(L, t^{a}, t^{b}\right)\right)=\prod_{i=1}^{n} d_{i}-P(a, b),
$$

and the second generalized footprint function of $I$ is

$$
\operatorname{fp}_{I}(d, 2)=\min \left\{P(a, b) \mid\left\{t^{a}, t^{b}\right\} \in \mathcal{M}_{\prec, d, 2}\right\} .
$$

Making the substitution $-\ell=\sum_{i=1}^{k}\left(d_{i}-1\right)-\sum_{i=1}^{s} a_{i}$ and using the fact that $\operatorname{fp}_{I(\mathbb{X})}(d, r)$ is less than or equal to $\delta_{\mathbb{X}}(d, r)$ (see Theorem 4.9) it suffices to show the inequalities

$$
P(a, b) \geq\left(\sum_{i=1}^{k+1}\left(d_{i}-a_{i}\right)-(k-1)-a_{s}-\sum_{i=k+2}^{n} a_{i}\right) d_{k+2} \cdots d_{n}-d_{k+3} \cdots d_{n},
$$

for $\left\{t^{a}, t^{b}\right\} \in \mathcal{M}_{\prec, d, 2}$ if $0 \leq k \leq s-3$, where $d_{k+3} \cdots d_{n}=1$ if $k=s-3$, and

$$
P(a, b) \geq \sum_{i=1}^{s-1}\left(d_{i}-a_{i}\right)-(s-3)-a_{s},
$$

for $\left\{t^{a}, t^{b}\right\} \in \mathcal{M}_{\prec, d, 2}$ if $k=s-2$. As $\left(a_{1}, \ldots, a_{n}\right)$ is not equal to $\left(b_{1}, \ldots, b_{n}\right)$, one has that either $\prod_{i=1}^{n} d_{i}-\prod_{i=1}^{n}\left(d_{i}-a_{i}\right) \geq 1$ or $\prod_{i=1}^{n} d_{i}-\prod_{i=1}^{n}\left(d_{i}-b_{i}\right) \geq 1$. If $a_{s} \geq 1$ or $b_{s} \geq 1\left(\right.$ resp. $\left.a_{s}=b_{s}=0\right)$, the inequality of Eq. (9.2) follows at once from [25, Proposition 5.7] (resp. Lemma 8.6). If $a_{s} \geq 1$ or $b_{s} \geq 1$ (resp. $a_{s}=b_{s}=0$ ), the inequality of Eq. (9.3) follows at once from [25, Proposition 5.7] (resp. Lemma 8.3). This completes the proof of the inequality " $\geq$ ".

The inequality " $\leq$ " follows directly from Lemma 9.1 .

Using Theorem 9.3 one recovers the following main result of [14].

Corollary 9.4. [14, Theorem 18] Let $K=\mathbb{F}_{q}$ be a finite field and let $\mathbb{T}$ be a projective torus in $\mathbb{P}^{s-1}$. If $d \geq 1$ and $s \geq 3$, then

$$
\delta_{\mathbb{T}}(d, 2)= \begin{cases}(q-1)^{s-(k+3)}[(q-1)(q-\ell)-1] & \text { if } 1 \leq d \leq \eta, \\ q-\ell & \text { if } \eta<d<\gamma, \\ 2 & \text { if } d \geq \gamma\end{cases}
$$


where $k$ and $\ell$ are the unique integers such that $d=k(q-2)+\ell, k \geq 0,1 \leq \ell \leq q-2$, $\eta=(q-2)(s-2)$ and $\gamma=(q-2)(s-1)$.

Another of our applications to coding theory is the following purely combinatorial formula for the second generalized Hamming weight of an affine cartesian code which is quite different from the corresponding formula of [1, Theorem 5.4].

Theorem 9.5. Let $\mathcal{P}_{d}$ be the set of all pairs $(a, b), a, b$ in $\mathbb{N}^{s}, a=\left(a_{1}, \ldots, a_{s}\right), b=\left(b_{1}, \ldots, b_{s}\right)$, such that $a \neq b, d=\sum_{i=1}^{s} a_{i}=\sum_{i=1}^{s} b_{i}, 1 \leq a_{i}, b_{i} \leq d_{i}-1$ for $i=1, \ldots, n, n:=s-1, a_{i} \neq 0$ and $b_{j} \neq 0$ for some $1 \leq i, j \leq n$. If $\mathbb{X}=\left[A_{1} \times \cdots \times A_{n} \times\{1\}\right] \subset \mathbb{P}^{n}$, with $A_{i} \subset \mathbb{F}_{q}, d_{i}=\left|A_{i}\right|$, and $2 \leq d_{1} \leq \cdots \leq d_{n}$, then

$$
\mathrm{fp}_{I(\mathbb{X})}(d, 2)=\delta_{\mathbb{X}}(d, 2)=\min \left\{P(a, b) \mid(a, b) \in \mathcal{P}_{d}\right\} \quad \text { for } \quad d \leq \sum_{i=1}^{n}\left(d_{i}-1\right),
$$

where $P(a, b)=\prod_{i=1}^{n}\left(d_{i}-a_{i}\right)+\prod_{i=1}^{n}\left(d_{i}-b_{i}\right)-\prod_{i=1}^{n} \min \left\{d_{i}-a_{i}, d_{i}-b_{i}\right\}$.

Proof. Let $\psi(d)$ be the formula for $\delta_{\mathbb{X}}(d, 2)$ given in Theorem 9.3 , Then using Eqs. (9.2) and Eqs. (9.3) one has $\psi(d) \leq \mathrm{fp}_{I(\mathbb{X})}(d, 2)$. By Theorem 4.9 one has $\mathrm{fp}_{I(\mathbb{X})}(d, r) \leq \delta_{\mathbb{X}}(d, r)$, and by Lemma 9.1 one has $\delta_{\mathbb{X}}(d, r) \leq \psi(d)$. Therefore

$$
\psi(d) \leq \mathrm{fp}_{I(\mathbb{X})}(d, 2) \leq \delta_{\mathbb{X}}(d, r) \leq \psi(d) .
$$

Thus we have equality everywhere and the result follows from Eq. (9.1).

Remark 9.6. Let $\psi(d)$ be the formula for $\delta_{\mathbb{X}}(d, 2)$ given in Theorem 9.3. Then

$$
\psi(d)=\min \left\{P(a, b) \mid(a, b) \in \mathcal{P}_{d}\right\}
$$

for $d \leq \sum_{i=1}^{n}\left(d_{i}-1\right)$. This equality is interesting in its own right.

\section{REFERENCES}

[1] P. Beelen and M. Datta, Generalized Hamming weights of affine Cartesian codes, Finite Fields Appl. 51 (2018), 130-145.

[2] W. Bruns and J. Herzog, Cohen-Macaulay Rings, Revised Edition, Cambridge University Press, 1997.

[3] C. Carvalho, On the second Hamming weight of some Reed-Muller type codes, Finite Fields Appl. 24 (2013), 88-94.

[4] C. Carvalho, V. G. Lopez Neumann and H. H. López, Projective nested cartesian codes, Bull. Braz. Math. Soc. (N.S.) 48 (2017), no. 2, 283-302.

[5] D. Cox, J. Little and D. O'Shea, Ideals, Varieties, and Algorithms, Springer-Verlag, 1992.

[6] M. Datta and S. Ghorpade, Number of solutions of systems of homogeneous polynomial equations over finite fields, Proc. Amer. Math. Soc. 145 (2017), no. 2, 525-541.

[7] I. M. Duursma, C. Rentería and H. Tapia-Recillas, Reed-Muller codes on complete intersections, Appl. Algebra Engrg. Comm. Comput. 11 (2001), no. 6, 455-462.

[8] D. Eisenbud, Commutative Algebra with a view toward Algebraic Geometry, Graduate Texts in Mathematics 150, Springer-Verlag, 1995.

[9] O. Geil, On the second weight of generalized Reed-Muller codes, Des. Codes Cryptogr. 48 (2008), 323-330.

[10] O. Geil, Evaluation codes from an affine variety code perspective, Advances in algebraic geometry codes, 153-180, Ser. Coding Theory Cryptol., 5, World Sci. Publ., Hackensack, NJ, 2008.

[11] O. Geil and T. Høholdt, Footprints or generalized Bezout's theorem, IEEE Trans. Inform. Theory 46 (2000), no. 2, 635-641.

[12] O. Geil and R. Pellikaan, On the structure of order domains, Finite Fields Appl. 8 (2002), no. 3, 369-396.

[13] O. Geil and C. Thomsen, Weighted Reed-Muller codes revisited, Des. Codes Cryptogr. 66 (2013), $195-220$.

[14] M. González-Sarabia, E. Camps, E. Sarmiento and R. H. Villarreal, The second generalized Hamming weight of some evaluation codes arising from a projective torus, Finite Fields Appl. 52 (2018), 370-394.

[15] M. González-Sarabia and C. Rentería, Generalized Hamming weights and some parameterized codes, Discrete Math. 339 (2016), 813-821. 
[16] M. González-Sarabia, C. Rentería and H. Tapia-Recillas, Reed-Muller-type codes over the Segre variety, Finite Fields Appl. 8 (2002), no. 4, 511-518.

[17] D. Grayson and M. Stillman, Macaulay2, 1996. Available via anonymous ftp from math.uiuc.edu.

[18] P. Heijnen and R. Pellikaan, Generalized Hamming weights of $q$-ary Reed-Muller codes, IEEE Trans. Inform. Theory 44 (1998), no. 1, 181-196.

[19] T. Helleseth, T. Kløve and J. Mykkelveit, The weight distribution of irreducible cyclic codes with block lengths $n_{1}\left(\left(q^{l}-1\right) / N\right)$, Discrete Math. 18 (1977), 179-211.

[20] T. Johnsen and H. Verdure, Generalized Hamming weights for almost affine codes, IEEE Trans. Inform. Theory 63 (2017), no. 4, 1941-1953.

[21] T. Kløve, The weight distribution of linear codes over $G F\left(q^{l}\right)$ having generator matrix over $G F(q)$, Discrete Math. 23 (1978), no. 2, 159-168.

[22] G. Lachaud, The parameters of projective Reed-Muller codes, Discrete Math. 81 (1990), no. 2, $217-221$.

[23] H. H. López, C. Rentería and R. H. Villarreal, Affine cartesian codes, Des. Codes Cryptogr. 71 (2014), no. $1,5-19$.

[24] F. J. MacWilliams and N. J. A. Sloane, The Theory of Error-correcting Codes, North-Holland, 1977.

[25] J. Martínez-Bernal, Y. Pitones and R. H. Villarreal, Minimum distance functions of graded ideals and ReedMuller-type codes, J. Pure Appl. Algebra 221 (2017), 251-275.

[26] J. Martínez-Bernal, Y. Pitones and R. H. Villarreal, Minimum distance functions of complete intersections, J. Algebra Appl., to appear.

[27] D. J. Mercier and R. Rolland, Polynômes homogènes qui s'annulent sur l'espace projectif $\mathbb{P}^{m}\left(\mathbb{F}_{q}\right)$, J. Pure Appl. Algebra 124 (1998), 227-240.

[28] L. Núñez-Betancourt, Y. Pitones and R. H. Villarreal, Footprint and minimum distance functions, Commun. Korean Math. Soc. 33 (2018), no. 1, 85-101.

[29] L. O'Carroll, F. Planas-Vilanova and R. H. Villarreal, Degree and algebraic properties of lattice and matrix ideals, SIAM J. Discrete Math. 28 (2014), no. 1, 394-427.

[30] W. Olaya-León and C. Granados-Pinzón, The second generalized Hamming weight of certain Castle codes, Des. Codes Cryptogr. 76 (2015), no. 1, 81-87.

[31] C. Rentería, A. Simis and R. H. Villarreal, Algebraic methods for parameterized codes and invariants of vanishing ideals over finite fields, Finite Fields Appl. 17 (2011), no. 1, 81-104.

[32] H. G. Schaathun and W. Willems, A lower bound on the weight hierarchies of product codes, Discrete Appl. Math. 128 (2003), no. 1, 251-261.

[33] A. Sørensen, Projective Reed-Muller codes, IEEE Trans. Inform. Theory 37 (1991), no. 6, 1567-1576.

[34] R. Stanley, Hilbert functions of graded algebras, Adv. Math. 28 (1978), 57-83.

[35] M. Tsfasman, S. Vladut and D. Nogin, Algebraic geometric codes: basic notions, Mathematical Surveys and Monographs 139, American Mathematical Society, Providence, RI, 2007.

[36] R. H. Villarreal, Monomial Algebras, Second Edition, Monographs and Research Notes in Mathematics, Chapman and Hall/CRC, 2015.

[37] V. K. Wei, Generalized Hamming weights for linear codes, IEEE Trans. Inform. Theory 37 (1991), no. 5, $1412-1418$.

[38] V. K. Wei and K. Yang, On the generalized Hamming weights of product codes, IEEE Trans. Inform. Theory 39 (1993), no. 5, 1709-1713.

[39] M. Yang, J. Lin, K. Feng and D. Lin, Generalized Hamming weights of irreducible cyclic codes, IEEE Trans. Inform. Theory 61 (2015), no. 9, 4905-4913. 
M.G. Sarabia. Instituto Politécnico Nacional, UPitta, Av. iPN No. 2580, Col. La Laguna Ticomán, Gustavo A. Madero C.P. 07340, Ciudad de México. Departamento de Ciencias Básicas

E-mail address: mgonzalezsa@ipn.mx

Departamento de Matemáticas, Centro de Investigación y de Estudios Avanzados del IPN, Apartado Postal 14-740, 07000 Mexico City, D.F.

E-mail address: jmb@math.cinvestav.mx

Departamento de Matemáticas, Centro de Investigación y de Estudios Avanzados del IPN, Apartado Postal 14-740, 07000 Mexico City, D.F.

E-mail address: vila@math.cinvestav.mx

Departamento de Matemáticas, Centro de Investigación y de Estudios Avanzados del IPN, Apartado Postal 14-740, 07000 Mexico City, D.F.

E-mail address: cevivares@math.cinvestav.mx 